TRANSACTIONS OF THE

AMERICAN MATHEMATICAL SOCIETY

Volume 351 , Number 10, Pages 4127-4171

S 0002-9947(99)02313-2

Article electronically published on July 1, 1999

\title{
NORM ESTIMATES AND REPRESENTATIONS FOR CALDERÓN-ZYGMUND OPERATORS USING AVERAGES OVER STARLIKE SETS
}

\author{
DAVID K. WATSON AND RICHARD L. WHEEDEN
}

ABSTRACT. We show that homogeneous singular integrals may be represented in terms of averages over starlike sets. This permits us to use the geometry of starlike sets to derive operator-specific weighted norm inequalities.

\section{INTRODUCTION AND MAIN RESULTS}

Homogeneous singular integrals on $\mathbf{R}^{d}, d \geq 2$, are given by

$$
T_{\Omega} f(x)=p . v . \int_{\mathbf{R}^{d}} f(x-y) \frac{\Omega(y)}{|y|^{d}} d y,
$$

where $\Omega$ is homogeneous of degree 0 and integrable on the unit sphere $\mathbf{S}^{d-1}$, with integral 0 . We may associate with this operator a set $S=S(\Omega)$ defined by

$$
\begin{aligned}
S & =\left\{x:|\Omega(x)| /|x|^{d} \geq 1\right\} \\
& =\left\{x: x=r \theta, \quad \theta \in \mathbf{S}^{d-1}, \quad 0 \leq r \leq|\Omega(\theta)|^{1 / d}\right\} .
\end{aligned}
$$

The set $S$ is starlike with respect to the origin, and using polar coordinates shows that $|S|=\frac{1}{d} \int_{\mathbf{S}^{d-1}}|\Omega(\theta)| d \theta$, so that $|S|<\infty$ iff $\Omega \in L^{1}\left(\mathbf{S}^{d-1}\right)$. In this paper we derive a new representation for $\mathrm{T}_{\Omega}$ in terms of averages over dilates of $S$. We then use this representation to derive weighted norm inequalities for $\mathrm{T}_{\Omega}$, with the geometry of $S$ being used to describe the weights.

In order to describe the representation, for $t>0$ let $t S=\{t x: x \in S\}$ and define averages

$$
\begin{aligned}
A_{t} f(x) & =\frac{1}{t^{d}} \int_{t S} f(x-y) \operatorname{sgn} \Omega(y) d y \\
& =\int_{S} f(x-t y) \operatorname{sgn} \Omega(y) d y,
\end{aligned}
$$

where $\operatorname{sgn} \Omega(y)$ is the complex sign of $\Omega$, i.e., $\operatorname{sgn} \Omega(y)=\Omega(y) /|\Omega(y)|$ when $\Omega(y) \neq$ $0, \infty$. We arbitrarily define $\operatorname{sgn} \Omega(y)$ to be 0 if $\Omega(y)=0$ or $\infty$, since this can be seen to have no effect on any of our formulas.

Our representation formula is contained in the following result.

Received by the editors December 30, 1996 and, in revsied form, December 15, 1997.

1991 Mathematics Subject Classification. Primary 42B20, 42B25.

Supported in part by NSF Grant DMS95-00799. 
Theorem 1.1. Either let $\Omega$ be odd with $\Omega \in L^{1}\left(\mathbf{S}^{d-1}\right)$, or else let $\Omega$ have integral 0 on $\mathbf{S}^{d-1}$ with $\Omega \in L \log L\left(\mathbf{S}^{d-1}\right)$, i.e.,

$$
\|\Omega\|_{L \log L\left(\mathbf{S}^{d-1}\right)}=\int_{\mathbf{S}^{d-1}}|\Omega(\theta)|\left(1+\log ^{+}|\Omega(\theta)|\right) d \theta<\infty .
$$

Then the integral $\int_{0}^{\infty} A_{t} f(x) d t / t=\lim _{\substack{t \rightarrow 0 \\ R \rightarrow \infty}} \int_{\epsilon}^{R} A_{t} f(x) d t / t$ converges absolutely for all $x \in \mathbf{R}^{d}$, uniformly in $x$, whenever $f, \widehat{f} \in L^{1}\left(\mathbf{R}^{d}\right)$ (in particular when $f \in \mathcal{S}$, the Schwartz class of smooth, rapidly decreasing functions on $\mathbf{R}^{d}$ ). The integral also converges in $L^{2}\left(\mathbf{R}^{d}\right)$ for $f \in L^{2}\left(\mathbf{R}^{d}\right)$ and defines a bounded operator on $L^{2}$.

Furthermore, if $\Omega$ is odd and $\Omega \in L^{1}\left(\mathbf{S}^{d-1}\right)$, then

$$
\mathrm{T}_{\Omega} f(x)=d \int_{0}^{\infty} A_{t} f(x) \frac{d t}{t}
$$

and if $\Omega$ has integral 0 on $\mathbf{S}^{d-1}$ and $\Omega \in L \log L\left(\mathbf{S}^{d-1}\right)$, then

$$
\mathrm{T}_{\Omega} f(x)=c_{\Omega} \cdot f(x)+d \int_{0}^{\infty} A_{t} f(x) \frac{d t}{t}
$$

where $c_{\Omega}=\frac{1}{d} \int_{\mathbf{S}^{d-1}} \Omega(\theta) \log |\Omega(\theta)| d \theta$. The identities (1.1) and (1.2) are valid in the sense of convergence in $L^{2}$ for $f \in L^{2}$. Also, if $f, \widehat{f} \in L^{1}\left(\mathbf{R}^{d}\right)$, then (1.1) holds pointwise for all $x \in \mathbf{R}^{d}$ and (1.2) holds pointwise almost everywhere, and (1.2) can be made to hold pointwise for all $x \in \mathbf{R}^{d}$ by redefining $f$ on a set of measure 0 so as to be continuous everywhere (which we can do since $\widehat{f} \in L^{1}$ ).

The requirements for the representation formulas (1.1) and (1.2) are the same minimal conditions of the method of rotations [CZ]. The representation (1.2) proves to be useful for obtaining weighted norm inequalities, particularly so when $\Omega$ is unbounded.

Let $\rho$ be the boundary function of $S$, i.e. $\rho(\theta)=|\Omega(\theta)|^{1 / d}$. As motivation for the theorem, let us proceed formally to write

$$
\begin{aligned}
\int_{0}^{\infty} A_{t} f(x) \frac{d t}{t} & =\int_{\mathbf{S}^{d-1}} \operatorname{sgn} \Omega(\theta) \int_{0}^{\rho(\theta)} \int_{0}^{\infty} f(x-\operatorname{tr} \theta) \frac{d t}{t} r^{d-1} d r d \theta \\
& =\int_{\mathbf{S}^{d-1}} \operatorname{sgn} \Omega(\theta)\left(\int_{0}^{\rho(\theta)} r^{d-1} d r\right)\left(\int_{0}^{\infty} f(x-t \theta) \frac{d t}{t}\right) d \theta \\
& =\frac{1}{d} \int_{\mathbf{S}^{d-1}} \int_{0}^{\infty} f(x-t \theta) \Omega(\theta) \frac{d t}{t} d \theta \\
& =\frac{1}{d} \operatorname{T}_{\Omega} f(x)
\end{aligned}
$$

with the second line arising by an unjustified interchange of the $t$ and $r$ integrals, followed by a change of variables in the $t$ integral so that it is independent of $r$. While (1.2) shows that this formula cannot always hold, it is correct if $f \in C_{0}^{\infty}$ and $x$ is outside the support of $f$. To see this, let $B$ be an upper bound for $|f|$ and let $0<\delta<R<\infty$ be such that supp $f \subseteq\{y: \delta<|x-y|<R\}$. Then the 
integral obtained by bringing absolute values inside the above integral converges, being bounded by

$$
\int_{\mathbf{S}^{d-1}} \int_{0}^{\rho(\theta)} r^{d-1} \int_{0}^{\infty} B \chi\{\delta<t r<R\} \frac{d t}{t} d r d \theta=B|S| \ln (R / \delta),
$$

so the formal operations in the preceding argument are justified.

The proof of Theorem 1.1 can be used to show that the operation mapping $f$ into the integral $\int_{0}^{\infty} A_{t} f(x) d t / t$ can be represented as convolution with a tempered distribution which is homogeneous of degree $-d$, and the preceding argument shows that this distribution can only differ from the kernel of $\frac{1}{d} \mathrm{~T}_{\Omega}$ by a point distribution at $\{0\}$. We see that by (1.2) the point distribution may in fact be a nonzero multiple of the Dirac measure.

When $\Omega \in L \log L\left(\mathbf{S}^{d-1}\right)$ with integral 0, we will use the representation (1.2) to prove weighted norm inequalities for $\mathrm{T}_{\Omega}$. The weights can be described fairly precisely in terms of a covering of $S$ by rectangles centered at the origin. A similar idea was used in $[\mathrm{CWW}]$ for fractional integral and maximal operators associated with a starlike set. We shall also give examples which show that in many cases the sufficient conditions are very close to being necessary.

These weighted inequalities are initially described in terms of a sequence of auxiliary operators whose description requires the following. Decompose the boundary function $\rho=|\Omega|^{1 / d}$ at heights $2^{m}, m \geq 0$, letting

$$
\begin{aligned}
\Theta_{0} & =\left\{\theta \in \mathbf{S}^{d-1}: \rho(\theta) \leq 1\right\}, \\
\Theta_{m} & =\left\{\theta \in \mathbf{S}^{d-1}: 2^{m-1}<\rho(\theta) \leq 2^{m}\right\}, \quad m>0 .
\end{aligned}
$$

Let $S_{m}$ be the starlike set whose boundary function is the restriction of $\rho$ to $\Theta_{m}$, so that $S$ is the disjoint (except for the origin ) union of $\left\{S_{m}\right\}_{m=0}^{\infty}$. Let $A_{t}^{m}$ be obtained from $A_{t}$ by replacing $S$ by $S_{m}$, that is,

$$
A_{t}^{m} f(x)=\frac{1}{t^{d}} \int_{t S_{m}} f(x-y) \operatorname{sgn} \Omega(y) d y .
$$

The auxiliary operators we will need are given by

$$
G_{j} f(x)=\sum_{m=0}^{\infty} \int_{2^{j-m-1}}^{2^{j+(N-1) m}} A_{t}^{m} f(x) \frac{d t}{t},
$$

where $N$ is a large integer which will vary with use. It is important to note that the operators $G_{j}$ are given by convolution with functions $g_{j}$ which scale by dilation, i.e., $g_{j}(x)=2^{-d j} g_{0}\left(2^{-j} x\right)$, and we will see (following (2.28)) that these functions are integrable, so that the operators $G_{j}$ are well-defined.

We will use the operators $G_{j}$ in our main result, which will be the basis for the remaining results of this section. As is customary, if $1<p<\infty$, we use $A_{p}$ to refer to Muckenhoupt's family of weights $w$ for which there is some constant $C$ so that

$$
\left(\frac{1}{|Q|} \int_{Q} w(x) d x\right)^{1 / p}\left(\frac{1}{|Q|} \int_{Q} w(x)^{-p^{\prime} / p} d x\right)^{1 / p^{\prime}} \leq C
$$

for all cubes $Q$, where (here and throughout the paper) $1 / p+1 / p^{\prime}=1$. 
Theorem 1.2. Let $\Omega \in L \log L\left(\mathbf{S}^{d-1}\right)$ with integral 0 . For $1<p<\infty$, suppose that $w \in A_{p}$ and that the square inequality

$$
\left\|\left\{\sum_{j}\left|G_{j} f_{j}\right|^{2}\right\}^{1 / 2}\right\|_{p, w} \leq C_{p, w}\left\|\left\{\sum_{j}\left|f_{j}\right|^{2}\right\}^{1 / 2}\right\|_{p, w}
$$

holds for a sufficiently large $N$ depending on $p$ and $w$. Then for $f \in \mathcal{S}$,

$$
\left\|\mathrm{T}_{\Omega} f\right\|_{p, w} \leq C_{p, w}^{\prime}\|f\|_{p, w}
$$

so that $\mathrm{T}_{\Omega}$ extends to a bounded operator on $L^{p}(w)$.

This result will be proven in $\S 2$ using a Littlewood-Paley decomposition. We shall discuss partial two-weight analogues of Theorem 1.2 in $\S 5$. This is our most general theorem. The square inequality requirement (1.4) might appear difficult to verify, but the remainder of $\S 2$ is devoted to deriving conditions on $w$ for which it holds. In $\S 3$ we will give examples which show that some of these sufficient conditions are very close to being necessary, which indicates how broad a result Theorem 1.2 is.

In order to formulate cases in which (1.4) holds, we will use the geometry associated with $S$ together with some results from [CWW] to derive appropriate conditions on $w$. These conditions are stated in terms of a collection of rectangles $\left\{R_{m, k}\right\}$ centered at the origin with orientations chosen so that $S$ is covered by $\bigcup_{m, k} R_{m, k}$ in an efficient manner. Here, $m \geq 0$ and $1 \leq k<k_{m}$ for $k_{m}$ possibly infinite. The index $m$ measures the longest edgelength of the rectangles; specifically, the longest side of each $R_{m, k}$ has length approximately $2^{m}$. The other significant property of the starlike cover is that for each $m \geq 0$, the set $\bigcup_{k} R_{m, k}$ contains $S_{m}$ and has measure $\leq C\left|S_{m}\right|$ for $C$ independent of $m$. We call the collection $\left\{R_{m, k}\right\}$ a stratified starlike cover of $S$. A more precise definition and the construction of such a cover will be given in section 4 . For a given rectangle $R_{m, k}$, we will use $\mathcal{B}\left(R_{m, k}\right)$ to denote the collection of translates and dilates of $R_{m, k}$, so that $\mathcal{B}\left(R_{m, k}\right)$ consists of all rectangles with the same orientation and eccentricity as $R_{m, k}$.

We shall prove the following result.

Theorem 1.3. Let $1<p<\infty$ and let $\Omega \in L \log L\left(\mathbf{S}^{d-1}\right)$. Let $\left\{R_{m, k}\right\}$ be a stratified starlike cover of the set $S=S(\Omega)=\left\{r \theta: 0 \leq r \leq|\Omega(\theta)|^{1 / d}, \theta \in \mathbf{S}^{d-1}\right\}$. If $w$ satisfies

$$
\left(\frac{1}{|R|} \int_{R} w(x) d x\right)^{1 / p}\left(\frac{1}{|R|} \int_{R} w(x)^{-p^{\prime} / p} d x\right)^{1 / p^{\prime}} \leq c
$$

for $R \in \mathcal{B}\left(R_{m, k}\right)$ for all $k$ and $m$, with $c$ independent of $R, k$ and $m$, then (1.4) holds. If in addition $\Omega$ has integral 0 over $\mathbf{S}^{d-1}$, then the corresponding singular integral operator $\mathrm{T}_{\Omega}$ satisfies

$$
\left\|\mathrm{T}_{\Omega} f\right\|_{p, w} \leq C\|\Omega\|_{L \log L\left(\mathbf{S}^{d-1}\right)}\|f\|_{p, w}
$$

for $f \in \mathcal{S}$, with $C$ independent of $\Omega$ and $f$.

In particular, we conclude that $\mathrm{T}_{\Omega}$ is bounded on $L^{p}$ for $1<p<\infty$ because (1.5) holds trivially for $w=1$, so we therefore recover the conclusion of the even kernel case of the method of rotations.

Theorem 1.3 does not specifically require $w \in A_{p}$, but this is automatic from the assumption (1.5) by fixing a rectangle $R_{m, k}$ and observing that we may include 
every cube $Q$ in a rectangle $R \in \mathcal{B}\left(R_{m, k}\right)$ in such a way as to keep the ratio $|Q| /|R|$ fixed.

We will also prove two more general results. The first of these concerns the case $p=2$ and can be stated as follows.

Theorem 1.4. For $\Omega \in L \log L\left(\mathbf{S}^{d-1}\right)$, let $\left\{R_{m, k}\right\}$ be a stratified starlike cover of $S(\Omega)$, and let $w$ satisfy the following estimate for $p=2$ :

$$
\left(\frac{1}{|R|} \int_{R} w(x) d x\right)^{1 / p}\left(\frac{1}{|R|} \int_{R} w(x)^{-p^{\prime} / p} d x\right)^{1 / p^{\prime}} \leq \frac{c_{m, k}}{\left|R_{m, k}\right|}
$$

for $R \in \mathcal{B}\left(R_{m, k}\right)$ for all $k$ and $m$. Then (1.4) holds for $p=2$ if

$$
\sum_{m, k}(m+1) c_{m, k}<\infty
$$

If in addition $\Omega$ has integral 0 over $\mathbf{S}^{d-1}$, then the corresponding singular integral operator $\mathrm{T}_{\Omega}$ satisfies (1.6) for $p=2$ and $f \in \mathcal{S}$.

We will show in $\S 4$ that if $c_{m, k}$ is a fixed multiple of $\left|R_{m, k}\right|$, then (1.8) will be a corollary of the fact that $\Omega \in L \log L\left(\mathbf{S}^{d-1}\right)$, so that Theorem 1.4 includes the $p=2$ case of Theorem 1.3. The next result of this section says that we need only a slight strengthening of (1.7) to extend the above result to $p \neq 2$.

Theorem 1.5. Suppose $\Omega \in L \log L\left(\mathbf{S}^{d-1}\right)$ has integral $0,1<p<\infty$, and that $\left\{R_{m, k}\right\}$ is a stratified starlike cover of $S(\Omega)$. Let $r>1$ and suppose that

$$
\begin{aligned}
& \left(\frac{1}{|R|} \int_{R} w\right)^{1 / p}\left(\frac{1}{|R|} \int_{R} w^{-r p^{\prime} / p}\right)^{1 / r p^{\prime}} \leq \frac{c_{m, k}}{\left|R_{m, k}\right|} \quad \text { if } 1<p \leq 2 \text {, or } \\
& \left(\frac{1}{|R|} \int_{R} w^{r}\right)^{1 / r p}\left(\frac{1}{|R|} \int_{R} w^{-p^{\prime} / p}\right)^{1 / p^{\prime}} \leq \frac{c_{m, k}}{\left|R_{m, k}\right|} \quad \text { if } 2 \leq p<\infty,
\end{aligned}
$$

for all $k, m$ and all $R \in \mathcal{B}\left(R_{m, k}\right)$, and that the constants $c_{m, k}$ satisfy (1.8). Then (1.4) and consequently (1.6) hold for $f \in \mathcal{S}$.

As we shall see, Theorem 1.5 includes all of Theorem 1.3. For $p=2$ and $r>$ 1 , either of the conditions (1.9), (1.10) is stronger than (1.7), and consequently Theorem 1.4 is a better result than Theorem 1.5 for the case $p=2$.

The following shows that Theorem 1.2 recovers and extends two fairly basic older results.

Theorem 1.6. (A) Let $1<r \leq \infty$, and suppose that $\Omega \in L^{r}\left(\mathbf{S}^{d-1}\right)$ and that $\Omega$ has integral 0 on $\mathbf{S}^{d-1}$. Then (1.4) holds for all $N$, and hence $\mathrm{T}_{\Omega}$ is bounded on $L^{p}(w)$, in the following cases:

(1) $r^{\prime} \leq p<\infty, p \neq 1$, and $w \in A_{p / r^{\prime}}$

(2) $1<p \leq r, p \neq \infty$, and $w^{-p^{\prime} / p} \in A_{p^{\prime} / r^{\prime}}$, or

(3) $1<p<\infty$, and $w^{r^{\prime}} \in A_{p}$.

(B) Suppose $\Omega \in L \log L\left(\mathbf{S}^{d-1}\right)$ has integral $0,1<p<\infty$, and $w(x)=|x|^{\beta}$ for $-1<\beta<p-1$. Then (1.4) holds for all $N$ and hence $\mathrm{T}_{\Omega}$ is bounded on $L^{p}(w)$. 
The weighted norm bounds in part (A) of this theorem were first proven for general $r$ in [KW1], under the stronger assumption that $\Omega$ also satisfies an $L^{r}$ Dini smoothness condition. In [W1], this smoothness condition was shown to be unnecessary by an argument using Fourier transform decay estimates to substitute for smoothness. Other proofs of the weighted inequalities in (A) are also given in $[\mathrm{D}],[\mathrm{W} 4]$. The norm inequalities in part (B) of Theorem 1.6 were proven in [MW], and the range of $\beta$ was shown to be sharp in [KW2].

Having stated our main results, let us outline the remainder of the paper. We will prove Theorems 1.1 and 1.2 in the next section. In $\S 3$ we will give some motivation for Theorems 1.3-1.5 by showing that the sufficient conditions are close to being necessary, and we will give the proofs of Theorems 1.3-1.6 in $\S 4$. Finally, in the last section, $\S 5$, we will give assorted results that did not fit naturally into the previous sections. Some of these results involve two-weight inequalities which we have only been able to show if $p \geq 2$ or if we require a greater degree of integrability of $\Omega$. We will also give another proof of part (A) of Theorem 1.6 which only uses the methods of this paper, as opposed to the proof given in $\S 4$, which uses some advanced results of [W4]. Lastly, $\S 5$ will show how the singular integral results of this paper also lead to corresponding results for the associated starlike maximal operator

$$
\mathrm{M}_{S} f(x)=\sup _{t>0} t^{-d} \chi_{t S} *|f|(x) .
$$

Some of these maximal operator results are different from those obtained in [CWW].

\section{Proofs of Theorems 1.1 And 1.2}

Proof of Theorem 1.1. Note that for each $t>0$ the operator $A_{t}$ is given by convolution with the function $a_{t}(\cdot)=t^{-d} \chi_{t S}(\cdot) \operatorname{sgn} \Omega(\cdot)$, which satisfies $\left\|a_{t}\right\|_{1} \leq|S|$. Consequently, $\left|\widehat{a}_{t}(\xi)\right| \leq|S|$ for all $\xi$ and we can write

$$
A_{t} f(x)=\left(\widehat{a}_{t} \widehat{f}\right)^{\vee}(x)=\int_{\mathbf{R}^{d}} e^{2 \pi i \xi \cdot x} \widehat{a}_{t}(\xi) \widehat{f}(\xi) d \xi,
$$

with this identity holding in $L^{2}$ if $f \in L^{2}$. Also, if $f, \widehat{f} \in L^{1}\left(\mathbf{R}^{d}\right)$, then $f \in L^{\infty}\left(\mathbf{R}^{d}\right)$ so $A_{t} f$ is bounded, continuous and integrable by Young's convolution theorem, and consequently (2.1) also holds for all $x \in \mathbf{R}^{d}$. (Here, we use the Fourier transform and inverse Fourier transform defined respectively by

$$
\widehat{f}(\xi)=\int_{\mathbf{R}^{d}} e^{-2 \pi i \xi \cdot x} f(x) d x, \quad g^{\vee}(x)=\int_{\mathbf{R}^{d}} e^{2 \pi i \xi \cdot x} g(\xi) d \xi,
$$

and their natural extensions to unitary operators on $L^{2}$, as in [SW2].) We shall prove the bound

$$
\int_{0}^{\infty}\left|\widehat{a}_{t}(\xi)\right| \frac{d t}{t} \leq D_{\Omega}
$$

which yields the claimed $L^{2}$ convergence of $\int_{0}^{\infty} A_{t} f d t / t$ by the Lebesgue dominated convergence theorem, and gives the pointwise convergence we seek when $f, \widehat{f} \in L^{1}$, since using (2.1) we see that

$$
\int_{0}^{\infty}\left|A_{t} f(x)\right| \frac{d t}{t} \leq \int_{\mathbf{R}^{d}} \int_{0}^{\infty}\left|\widehat{a}_{t}(\xi)\right| \frac{d t}{t}|\widehat{f}(\xi)| d \xi \leq D_{\Omega}\|\widehat{f}\|_{1} .
$$


Also, if we integrate (2.1), then by (2.3) we may use Fubini's theorem to obtain

$$
\int_{0}^{\infty} A_{t} f(x) \frac{d t}{t}=(m \widehat{f})^{\vee}(x)
$$

for all $x$ if $f, \widehat{f} \in L^{1}$, where

$$
m(\xi)=\int_{0}^{\infty} \widehat{a}_{t}(\xi) \frac{d t}{t} .
$$

To prove $(2.2)$, using polar coordinates we have

$$
\widehat{a}_{t}(\xi)=\int_{\mathbf{S}^{d-1}} \operatorname{sgn} \Omega(\theta) \int_{0}^{\rho(\theta)} e^{-2 \pi i t r \xi \cdot \theta} r^{d-1} d r d \theta
$$

where we recall that $\rho(\theta)=|\Omega(\theta)|^{1 / d}$. For the case of odd $\Omega \in L^{1}\left(\mathbf{S}^{d-1}\right)$ we can replace $\exp \{i \cdot\}$ in this formula with $-i \sin \{\cdot\}$, and since $|\sin u| \leq|u|$, then letting $v(\theta)=|\xi \cdot \theta| \rho(\theta)$ we have the absolute value estimate

$$
\left|\int_{0}^{\rho(\theta)} \sin (2 \pi t r \xi \cdot \theta) r^{d-1} d r\right| \leq c_{d} t v(\theta)|\Omega(\theta)| .
$$

Also, writing $\sin (r u)=-\frac{1}{u} \frac{d}{d r} \cos (r u)$ and integrating by parts, we have

$$
\left|\int_{0}^{\rho(\theta)} \sin (2 \pi t r \xi \cdot \theta) r^{d-1} d r\right| \leq \frac{c_{d}|\Omega(\theta)|}{t v(\theta)}
$$

for the same choice of $v$. Thus

$$
\begin{aligned}
& \int_{0}^{\infty}\left|\widehat{a}_{t}(\xi)\right| \frac{d t}{t} \leq c_{d} \int_{0}^{\infty} \int_{\mathbf{S}^{d-1}}|\Omega(\theta)| \min \left\{t v(\theta), \frac{1}{t v(\theta)}\right\} d \theta \frac{d t}{t} \\
& =c_{d} \int_{\mathbf{S}^{d-1}}|\Omega(\theta)| \int_{0}^{\infty} \min \left\{t, \frac{1}{t}\right\} \frac{d t}{t} d \theta=2 c_{d} \int_{\mathbf{S}^{d-1}}|\Omega(\theta)| d \theta .
\end{aligned}
$$

The desired convergence in the case of general $\Omega \in L \log L\left(\mathbf{S}^{d-1}\right)$ with integral 0 is more difficult. Using the cancellation of $\Omega$, we see that $a_{t}$ has integral 0 :

$$
\int_{\mathbf{R}^{d}} a_{t}(y) d y=\frac{1}{t^{d}} \int_{\mathbf{S}^{d-1}} \operatorname{sgn} \Omega(\theta) \int_{0}^{t \rho(\theta)} r^{d-1} d r d \theta=\frac{1}{d} \int_{\mathbf{S}^{d-1}} \Omega(\theta) d \theta=0 .
$$

Therefore

$$
\widehat{a}_{t}(\xi)=\int_{\mathbf{S}^{d-1}} \operatorname{sgn} \Omega(\theta) \int_{0}^{\rho(\theta)}\left[e^{-2 \pi i t r \xi \cdot \theta}-1\right] r^{d-1} d r d \theta .
$$

By arguing essentially as for (2.5) and (2.6) (using, e.g., the fact that $\left|e^{i u}-1\right|$ is bounded by both $|u|$ and 2), we get

$$
\begin{gathered}
\left|\int_{0}^{\rho(\theta)}\left[e^{-2 \pi i t r \xi \cdot \theta}-1\right] r^{d-1} d r\right| \leq c_{d}|\Omega(\theta)| \min \{1, t v(\theta)\} \\
\quad\left|\int_{0}^{\rho(\theta)} e^{-2 \pi i t r \xi \cdot \theta} r^{d-1} d r\right| \leq c_{d}|\Omega(\theta)| \min \left\{1, \frac{1}{t v(\theta)}\right\}
\end{gathered}
$$


for the same choice of $v(\theta)$. Letting $\chi(t)$ denote the characteristic function of $[0,1]$, and using (2.8), we can write

$$
\begin{aligned}
\int_{0}^{\infty}\left|\widehat{a}_{t}(\xi)\right| \frac{d t}{t} & =\int_{0}^{\infty}\left|\int_{\mathbf{S}^{d-1}} \operatorname{sgn} \Omega(\theta) \int_{0}^{\rho(\theta)}\left[e^{-2 \pi i t r \xi \cdot \theta}-\chi(|\xi| t)\right] r^{d-1} d r d \theta\right| \frac{d t}{t} \\
& =\quad A+B,
\end{aligned}
$$

where $A$ is the part of the integral for $0<t<1 /|\xi|$ and $B$ is the remaining part. Then

$$
\begin{aligned}
& A \leq c_{d} \int_{0}^{1 /|\xi|} \int_{\mathbf{S}^{d-1}}|\Omega(\theta)| \min \{1, t v(\theta)\} d \theta \frac{d t}{t} \\
& B \leq c_{d} \int_{1 /|\xi|}^{\infty} \int_{\mathbf{S}^{d-1}}|\Omega(\theta)| \min \left\{1, \frac{1}{t v(\theta)}\right\} d \theta \frac{d t}{t}
\end{aligned}
$$

using (2.9), (2.10) respectively. Interchange the order of integration and split the $t$ integral into integrals over intervals $(0, \alpha(\theta))$ and $(\alpha(\theta), 1 /|\xi|)$, where $\alpha(\theta)=$ $\min \{1 /|\xi|, 1 / v(\theta)\}$, to see that $A$ is bounded by

$$
c_{d} \int_{\mathbf{S}^{d-1}}|\Omega(\theta)|\left(\alpha(\theta) v(\theta)+\ln ^{+} \frac{v(\theta)}{|\xi|}\right) d \theta \leq c_{d} \int_{\mathbf{S}^{d-1}}|\Omega(\theta)|\left(1+\frac{1}{d} \ln ^{+}|\Omega(\theta)|\right) d \theta
$$

uniformly in $\xi$. (The last inequality uses $\alpha v \leq 1$ and $|\xi \cdot \theta| \leq|\xi|$.) Next, let $\beta(\theta)=$ $\max \{1 /|\xi|, 1 / v(\theta)\}$, interchange the order of integration and split the interval of $t$ integration into the intervals $(1 /|\xi|, \beta(\theta))$ and $(\beta(\theta), \infty)$, to see that $B$ is bounded by a geometric multiple of

$$
\begin{aligned}
\int_{\mathbf{S}^{d-1}}|\Omega(\theta)|\left(1+\ln ^{+} \frac{|\xi|}{v(\theta)}\right) d \theta & \leq\|\Omega\|_{L \log L\left(\mathbf{S}^{d-1}\right)}+\int_{\mathbf{S}^{d-1}}|\Omega(\theta)| \ln \frac{|\xi|}{|\xi \cdot \theta|} d \theta \\
& \leq c_{d}\left(1+\|\Omega\|_{L \log L\left(\mathbf{S}^{d-1}\right)}\right)
\end{aligned}
$$

uniformly in $\xi$. To see the last line, observe that the functions $\phi(t)=e^{t}-1$, $\psi(t)=\ln (t+1)$ vanish at 0 , are nonnegative, increasing and inverse to each other for $t \geq 0$, giving the inequality

$$
a b \leq \int_{0}^{a} \phi(t) d t+\int_{0}^{b} \psi(t) d t \leq e^{a}+b \ln (b+1)
$$

for all $a, b \geq 0$ by Young's generalization of Hölder's inequality e.g., [Z, vol. 1, p. 16]. Letting $\xi^{\prime}=\xi /|\xi|$, the last line of (2.11) then follows by taking $a=\frac{1}{2} \ln \frac{1}{\left|\xi^{\prime} \cdot \theta\right|}$, $b=2|\Omega(\theta)|$ and integrating in $\theta$, observing that $\exp \left(\frac{1}{2} \ln \frac{1}{\left|\xi^{\prime} \cdot \theta\right|}\right)=\left|\xi^{\prime} \cdot \theta\right|^{-1 / 2}$ is integrable uniformly in $\xi$.

We are now left with the task of showing the identities (1.1) and (1.2). We will prove these identities by showing the corresponding identity of the Fourier 
multipliers. Whenever they are defined, let

$$
\begin{aligned}
& m_{o}(\xi)=-i \frac{\pi}{2} \int_{\mathbf{S}^{d-1}} \Omega(\theta) \operatorname{sgn}(\xi \cdot \theta) d \theta \\
& m_{e}(\xi)=\int_{\mathbf{S}^{d-1}} \Omega(\theta) \log \frac{1}{\left|\xi^{\prime} \cdot \theta\right|} d \theta, \quad \xi^{\prime}=\xi /|\xi|
\end{aligned}
$$

Also let $m_{\Omega}(\xi)$ be the multiplier of $\mathrm{T}_{\Omega}$, that is $\left(\mathrm{T}_{\Omega} f\right)^{\uparrow}(\xi)=m_{\Omega}(\xi) \widehat{f}(\xi)$. We know (e.g. [CZ] and also [S]) that $m_{\Omega}=m_{o}+m_{e}$, that $m_{\Omega}=m_{o}$ for odd $\Omega \in L^{1}\left(\mathbf{S}^{d-1}\right)$ and $m_{\Omega}=m_{e}$ for even $\Omega \in L \log L\left(\mathbf{S}^{d-1}\right)$ with integral 0 , and that $m_{\Omega}$ is bounded for $\xi \neq 0$ for these cases. If $m$ is the multiplier in (2.4), then (1.1) will follow once we show $m(\xi)=m_{o}(\xi) / d$ when $\Omega$ is integrable and odd, and (1.2) will follow once we show $m(\xi)=m_{\Omega}(\xi) / d-c_{\Omega} / d$ for $\Omega \in L \log L\left(\mathbf{S}^{d-1}\right)$ with integral 0 . This plainly gives (1.1), (1.2) immediately in the $L^{2}$ sense for $f \in L^{2}$. Further, if $f, \widehat{f} \in L^{1}$ and if we redefine $f$ on a set of measure 0 so that it is everywhere continuous, then the multiplier identities show that (1.1), (1.2) also hold in the pointwise sense for all $x$, since the same kind of truncation and limiting argument by which we are able to conclude that $\int_{0}^{\infty} A_{t} f(x) d t / t=(m \widehat{f})^{\vee}(x)$ for all $x$ can be used to show a similar identity for $T_{\Omega}$. In fact, suppose that $f, \widehat{f} \in L^{1}$ and that $\Omega$ is as in Theorem 1.1, and let

$$
K_{\epsilon R}(x)=\frac{\Omega(x)}{|x|^{d}} \chi\{\epsilon<|x|<R\}, \quad 0<\epsilon<R<\infty .
$$

Then $K_{\epsilon R}$ is integrable, telling us that $K_{\epsilon R} * f$ is integrable, bounded and continuous, in which case

$$
K_{\epsilon R} * f(x)=\left(\widehat{K}_{\epsilon R} \widehat{f}\right)^{\vee}(x)
$$

everywhere. But (see [CZ], [S]) we know that $\widehat{K}_{\epsilon R}(\xi)$ is uniformly bounded for $0<\epsilon<R<\infty$ and $\xi \in \mathbf{R}^{d}$, and that $\widehat{K}_{\epsilon R}(\xi) \rightarrow m_{\Omega}(\xi)$ for $\xi \neq 0$ as $\epsilon \rightarrow 0$, $R \rightarrow \infty$, which gives

$$
\mathrm{T}_{\Omega} f(x)=\lim _{\substack{\epsilon \rightarrow 0 \\ R \rightarrow \infty}} K_{\epsilon R} * f(x)=\left(m_{\Omega} \widehat{f}\right)^{\vee}(x)
$$

for all $x \in \mathbf{R}^{d}$ by the Lebesgue dominated convergence theorem.

We first prove the multiplier identity when $\Omega$ is odd. Recall that in this case $\widehat{a}_{t}(\xi)$ can be written as a sine integral. The improper integral identity

$$
\int_{0}^{\infty} \sin (t u) \frac{d t}{t}=\frac{\pi}{2} \operatorname{sgn} u
$$

is well-known, and integrating by parts shows that

$$
\left|\int_{\alpha}^{\beta} \sin (t u) \frac{d t}{t}\right| \leq \frac{c}{\alpha|u|}
$$

when $0<\alpha<\beta<\infty$. This tells us that

$$
\left|\frac{\pi}{2} \operatorname{sgn} u-\int_{0}^{\alpha} \sin (t u) \frac{d t}{t}\right| \leq \frac{c}{\alpha|u|}
$$


whenever $\alpha>0, u \neq 0$. The absolute convergence argument in the proof of (2.7) shows that we may interchange the $t$ and $\theta$ integrals to write

$$
\begin{aligned}
\int_{0}^{\infty} \widehat{a}_{t}(\xi) \frac{d t}{t} & =-i \int_{\mathbf{S}^{d-1}} \operatorname{sgn} \Omega(\theta) \int_{0}^{\infty} \int_{0}^{\rho(\theta)} \sin (2 \pi t r \xi \cdot \theta) r^{d-1} d r \frac{d t}{t} d \theta \\
& =\lim _{R \rightarrow \infty}-i \int_{\mathbf{S}^{d-1}} \operatorname{sgn} \Omega(\theta) \int_{0}^{\rho(\theta)} r^{d-1} \int_{0}^{R / v(\theta)} \sin (2 \pi t r \xi \cdot \theta) \frac{d t}{t} d r d \theta
\end{aligned}
$$

In the last line we wrote the $t$ integral as a limit, which permitted us to interchange the $t$ and $r$ integrals within the limit. By (2.12), the $t$ integral inside the limit on the last line is $\frac{\pi}{2} \operatorname{sgn}(\xi \cdot \theta)$ up to a difference term, which tells us that the quantity within the limit is $m_{o}(\xi) / d$ up to the corresponding $r, \theta$ integral of the difference term. By (2.12), this difference term integral is bounded by

$$
c \int_{\mathbf{S}^{d-1}} \int_{0}^{\rho(\theta)} r^{d-1} \frac{\rho(\theta)}{R r} d r d \theta \leq \frac{c^{\prime}}{R} \int_{\mathbf{S}^{d-1}}|\Omega(\theta)| d \theta,
$$

which plainly vanishes as $R \rightarrow \infty$. Consequently, we have

$$
\int_{0}^{\infty} \widehat{a}_{t}(\xi) \frac{d t}{t}=\frac{m_{o}(\xi)}{d}
$$

which is what we needed to show in the odd kernel case.

We now turn to the case $\Omega \in L \log L\left(\mathbf{S}^{d-1}\right)$ with integral 0 on $\mathbf{S}^{d-1}$. Here $\widehat{a}_{t}$ can be written as the sum of the sine and cosine integrals

$$
\begin{aligned}
& \widehat{a}_{t}^{\sin }(\xi)=-i \int_{\mathbf{S}^{d-1}} \operatorname{sgn} \Omega(\theta) \int_{0}^{\rho(\theta)} \sin (2 \pi t r \xi \cdot \theta) r^{d-1} d r d \theta, \\
& \widehat{a}_{t}^{\cos }(\xi)=\int_{\mathbf{S}^{d-1}} \operatorname{sgn} \Omega(\theta) \int_{0}^{\rho(\theta)} \cos (2 \pi t r \xi \cdot \theta) r^{d-1} d r d \theta,
\end{aligned}
$$

and we have just shown that

$$
\int_{0}^{\infty} \widehat{a}_{t}^{\sin }(\xi) \frac{d t}{t}=\frac{m_{o}(\xi)}{d}
$$

so we will be done if we show that

$$
\int_{0}^{\infty} \widehat{a}_{t}^{\cos }(\xi) \frac{d t}{t}=\frac{m_{e}(\xi)}{d}-\frac{c_{\Omega}}{d}
$$

To do this, recall that $\chi(t)$ is the characteristic function of the interval $[0,1]$ and that $v(\theta)=|\xi \cdot \theta| \rho(\theta)$. Since $a_{t}$ has integral 0 for each $t$, and since cos is an even function, we have

$$
\begin{aligned}
\int_{0}^{\infty} \widehat{a}_{t}^{\cos }(\xi) \frac{d t}{t} & =\int_{\mathbf{S}^{d-1}} \operatorname{sgn} \Omega(\theta) \int_{0}^{\infty} \int_{0}^{\rho(\theta)}[\cos (2 \pi t r|\xi \cdot \theta|)-\chi(|\xi| t)] r^{d-1} d r \frac{d t}{t} d \theta \\
& =I+I I
\end{aligned}
$$


where

$$
I=\int_{\mathbf{S}^{d-1}} \operatorname{sgn} \Omega(\theta) \int_{0}^{\infty} \int_{0}^{\rho(\theta)}[\chi(t v(\theta))-\chi(|\xi| t)] r^{d-1} d r \frac{d t}{t} d \theta
$$

and $I I$ is the remaining part (we are again justified in interchanging the $t$ and $\theta$ integrals because we were justified in doing so for the full multiplier integral and for the sine part of that integral by the proof of (2.2)). Bringing absolute values inside $I$, we see that the resulting integral converges absolutely for $\xi \neq 0$, being bounded by

$$
\frac{1}{d} \int_{\mathbf{S}^{d-1}}|\Omega(\theta)|\left|\ln \frac{|\xi|}{v(\theta)}\right| d \theta \leq \frac{1}{d} \int_{\mathbf{S}^{d-1}}|\Omega(\theta)|\left(\ln \frac{1}{\left|\xi^{\prime} \cdot \theta\right|}+\frac{1}{d}|\ln | \Omega(\theta)||\right) d \theta,
$$

which we can show is bounded by a constant independent of $\xi$ by arguing as for (2.11). It is therefore permissible to bring the $t$ integral all the way inside to see that

$$
\begin{aligned}
I & =\frac{1}{d} \int_{\mathbf{S}^{d-1}} \Omega(\theta) \ln \frac{1}{\left|\xi^{\prime} \cdot \theta\right| \rho(\theta)} d \theta \\
& =\frac{1}{d} \int_{\mathbf{S}^{d-1}} \Omega(\theta) \ln \frac{1}{\left|\xi^{\prime} \cdot \theta\right|} d \theta-\frac{1}{d^{2}} \int_{\mathbf{S}^{d-1}} \Omega(\theta) \ln |\Omega(\theta)| d \theta \\
& =m_{e}(\xi)-\frac{1}{d} c_{\Omega} .
\end{aligned}
$$

We are therefore done if we can show that the term $I I$ vanishes. But

$$
\begin{aligned}
I I & =\lim _{\substack{\epsilon \rightarrow 0 \\
R \rightarrow \infty}} \int_{\mathbf{S}^{d-1}} \operatorname{sgn} \Omega(\theta) \int_{\frac{\epsilon}{v(\theta)}}^{\frac{R}{v(\theta)}} \int_{0}^{\rho(\theta)}[\cos (2 \pi t r|\xi \cdot \theta|)-\chi(t v(\theta))] r^{d-1} d r \frac{d t}{t} d \theta \\
& =\lim _{\substack{\epsilon \rightarrow 0 \\
R \rightarrow \infty}} \int_{\mathbf{S}^{d-1}} \operatorname{sgn} \Omega(\theta) \int_{0}^{\rho(\theta)} r^{d-1} \int_{\epsilon / v(\theta)}^{R / v(\theta)} \cos (2 \pi t r|\xi \cdot \theta|) \frac{d t}{t} d r d \theta \\
& =\lim _{\substack{\epsilon \rightarrow 0 \\
R \rightarrow \infty}} \int_{\mathbf{S}^{d-1}} \operatorname{sgn} \Omega(\theta) \int_{0}^{\rho(\theta)} r^{d-1} \int_{\epsilon r / \rho(\theta)}^{R r / \rho(\theta)} \cos (2 \pi t) \frac{d t}{t} d r d \theta .
\end{aligned}
$$

The second line follows by interchanging the $t$ and $r$ integrals inside the limit because

$$
\int_{\mathbf{S}^{d-1}} \operatorname{sgn} \Omega(\theta) \int_{0}^{\rho(\theta)} r^{d-1} \int_{\epsilon / v(\theta)}^{R / v(\theta)} \frac{d t}{t} d r d \theta=\frac{1}{d} \ln \frac{R}{\epsilon} \int_{\mathbf{S}^{d-1}} \Omega(\theta) d \theta=0,
$$

which allows us to drop the subtracted expression appearing in the first line of (2.13), as its integral is just the above expression with $R=1$. The last line in (2.13) then follows by a substitution in the $t$ integral. But whenever $u(t)$ is a 
suitable function, interchanging the order of integration shows that

$$
\begin{aligned}
\int_{0}^{\rho(\theta)} r^{d-1} \int_{\epsilon r / \rho(\theta)}^{R r / \rho(\theta)} u(t) \frac{d t}{t} d r= & \int_{0}^{\epsilon} u(t) \int_{\rho(\theta) t / R}^{\rho(\theta) t / \epsilon} r^{d-1} d r \frac{d t}{t} \\
& +\int_{\epsilon}^{R} u(t) \int_{\rho(\theta) t / R}^{\rho(\theta)} r^{d-1} d r \frac{d t}{t} \\
= & |\Omega(\theta)| \int_{0}^{R} u(t) h_{\epsilon, R}(t) d r \frac{d t}{t}
\end{aligned}
$$

for $h_{\epsilon, R}(t)=t^{d}\left(1 / \epsilon^{d}-1 / R^{d}\right) / d$ if $0<t<\epsilon$ and $h_{\epsilon, R}(t)=\left(1-(t / R)^{d}\right) / d$ if $\epsilon<t<R$. The integral in the bottom line is clearly finite for $0<\epsilon<R<\infty$ with $u(t)=\cos 2 \pi t$, and so we see that the integral in the limit on the bottom line of (2.13) is 0 , being a multiple of the integral of $\Omega$. Since $I I=0$, we are done.

\section{LitTlewood-PALEy ARgument For $\mathrm{T}_{\Omega}$}

Proof of Theorem 1.2. The proof requires the extensive use of weighted LittlewoodPaley theory. Define a Littlewood-Paley decomposition

$$
\left.\left(Q_{j} f\right)\right\urcorner(\xi)=\Psi\left(2^{j}|\xi|\right) \widehat{f}(\xi),
$$

with $\Psi \in C_{0}^{\infty}((1 / 2,2))$ a nonnegative smooth function satisfying $\sum_{j \in Z} \Psi^{2}\left(2^{j} t\right)=1$ for $t>0$. Then Plancherel's theorem shows that $\sum_{j \in Z} Q_{j}^{2}=\operatorname{Id}$ on $L^{2}$, and this identity also holds in several other senses that we will describe later. One of those senses is in $L^{p}(w)$ norm for $1<p<\infty, w \in A_{p}$, in which case we also have the following weighted inequalities: given $1<p<\infty$ and $w \in A_{p}$, there are positive constants $c_{p, w}, C_{p, w}, C_{p, w}^{\prime}$, depending only on $p$ and the $A_{p}$ constant of $w$, such that

$$
\begin{gathered}
c_{p, w}\|f\|_{p, w} \leq\left\|\left\{\sum_{j}\left|Q_{j} f\right|^{2}\right\}^{1 / 2}\right\|_{p, w} \leq C_{p, w}\|f\|_{p, w}, \\
\left\|\sum_{j} Q_{j} f_{j}\right\|_{p, w} \leq C_{p, w}^{\prime}\left\|\left\{\sum_{j}\left|Q_{j} f_{j}\right|^{2}\right\}^{1 / 2}\right\|_{p, w} .
\end{gathered}
$$

In $\S 5$ we will need two-weight analogues of these inequalities. To get these analogues, first note that in particular the above inequalities hold for all $p>1$ if $w \in A_{1}$, i.e., for $w$ such that $M w \leq C w$ a.e., where $M$ is the Hardy-Littlewood maximal operator and the constant depends only on the $A_{1}$ constant of $w$. Now if we define $M_{r} f(x)=\left\{M\left(|f|^{r}\right)\right\}^{1 / r}$, the Coifman-Rochberg inequality says that for $r>1$

$$
M M_{r} f(x) \leq C_{r} M_{r} f(x) \text { a.e. } x,
$$

i.e., if $r>1$ and $M_{r} f$ is not infinite on a set of positive measure, then $M_{r} f$ is an $A_{1}$ weight with constant independent of $f$. From this and (2.14), (2.15), we immediately obtain the corresponding two-weight inequalities

$$
\begin{gathered}
\left\|\left\{\sum_{j}\left|Q_{j} f\right|^{2}\right\}^{1 / 2}\right\|_{L^{p}(v)} \leq C_{p, r}\|f\|_{L^{p}\left(M_{r} v\right)}, \\
\left\|\sum_{j} Q_{j} f_{j}\right\|_{L^{p}(v)} \leq C_{p, r}^{\prime}\left\|\left\{\sum_{j}\left|Q_{j} f_{j}\right|^{2}\right\}^{1 / 2}\right\|_{L^{p}\left(M_{r} v\right)},
\end{gathered}
$$


for $1<p<\infty, r>1$, and for all weights $v$, with constants $C_{p, r}, C_{p, r}^{\prime}$ depending only on $p$ and $r$. The inequalities (2.14)-(2.18) also hold with $Q_{j}^{2}$ replacing $Q_{j}$ at every occurrence in each equation. In (2.15) and (2.18), the inequality remains true if we delete the $Q_{j}$ on the right side, and we may also delete $Q_{j}^{2}$ on the right side of the variants of (2.15) and (2.18) having $Q_{j}^{2}$ in place of $Q_{j}$.

Basic weighted Littlewood-Paley theory is contained in $[\mathrm{K}]$. However, since some of the forms we use, including the variants described above, do not appear to be explicitly proven anywhere, we shall give a thumbnail sketch of the proofs of weighted Littlewood-Paley theory as it will be used here. Observe that if $\psi_{j}(x)$ is the Schwartz function given by $\widehat{\psi}_{j}(\xi)=\Psi\left(2^{j}|\xi|\right)$, then $Q_{j} f=f * \psi_{j}$ with

$$
\left|\psi_{j}(x)\right| \leq C_{m} \frac{2^{-d j}}{\left(1+\left|2^{-j} x\right|\right)^{m}}, \quad\left|\nabla \psi_{j}(x)\right| \leq C_{m} \frac{2^{-(d+1) j}}{\left(1+\left|2^{-j} x\right|\right)^{m}},
$$

for all $m>0$, uniformly in $j$. The use of this together with the support of the Fourier transform of $\psi_{j}$ tells us that if $K$ is a kernel of the form

$$
K(x)=\sum_{j=-m}^{n} \pm \psi_{j}(x)
$$

then $K$ satisfies $|K(x)| \leq D|x|^{-d},|\nabla K(x)| \leq D|x|^{-(d+1)},|\widehat{K}(\xi)| \leq D$, with $D$ independent of $m, n$ and choice of signs \pm , and as a consequence of weighted Calderón-Zygmund theory, we get the bounds

$$
\|K * f\|_{p, w} \leq C_{p, w}\|f\|_{p, w}, \quad 1<p<\infty, \quad w \in A_{p},
$$

with similar uniformity. From this, the last inequality in (2.14) follows by a standard Rademacher function argument. We get the corresponding inequality with $Q_{j}^{2}$ replacing $Q_{j}$ by observing that the above argument holds upon replacing $\psi_{j}$ with the function whose Fourier transform is $\Psi\left(2^{j}|\xi|\right)^{2}$.

Now recall that $L^{p^{\prime}}\left(w^{-p^{\prime} / p}\right)$ is the dual of $L^{p}(w)$ under the standard pairing $\langle f, g\rangle=\int f \bar{g}$ and that $w^{-p^{\prime} / p} \in A_{p^{\prime}}$ iff $w \in A_{p}$. Since the operators $Q_{j}$ are self-adjoint, if $f \in L^{2} \cap L^{p}(w)$ and $g \in L^{2} \cap L^{p^{\prime}}\left(w^{-p^{\prime} / p}\right)$, then

$$
\int_{\mathbf{R}^{d}} f(x) \overline{g(x)} d x=\sum_{j} \int_{\mathbf{R}^{d}} \Psi\left(2^{j}|\xi|\right)^{2} \widehat{f}(\xi) \overline{\widehat{g}(\xi)} d \xi=\sum_{j} \int_{\mathbf{R}^{d}} Q_{j} f(x) \overline{Q_{j} g(x)} d x .
$$

This results in

$$
\begin{aligned}
\left|\int f \bar{g}\right| & \leq\left\|\left\{\sum_{j}\left|Q_{j} f\right|^{2}\right\}^{1 / 2}\right\|_{p, w}\left\|\left\{\sum_{j}\left|Q_{j} g\right|^{2}\right\}^{1 / 2}\right\|_{p^{\prime}, w^{-p^{\prime} / p}} \\
& \leq C_{p^{\prime}, w^{-p^{\prime} / p}}\left\|\left\{\sum_{j}\left|Q_{j} f\right|^{2}\right\}^{1 / 2}\right\|_{p, w}\|g\|_{p^{\prime}, w^{-p^{\prime} / p}}
\end{aligned}
$$

where the last estimate follows from the last inequality in (2.14), which we have already proven. By duality and a density argument, this gives the first inequality in (2.14).

Observe that the last inequality in (2.14) holds if we replace $Q_{j}$ with operators $\widetilde{Q}_{j}$ which are defined in the same way, but with $\Psi$ replaced by a function $\widetilde{\Psi} \in$ $C_{0}^{\infty}((1 / 4,4))$ satisfying $\widetilde{\Psi}(t)=1$ for $1 / 2 \leq t \leq 2$. Consequently, the first inequality 
in (2.14) holds if we replace $Q_{j} f$ with $Q_{j}^{2} f$ by arguing as above but beginning instead with the identity

$$
\int f \bar{g}=\sum_{j} \int Q_{j}^{2} f{\widetilde{Q_{j}}}
$$

which follows upon taking Fourier transforms since $\Psi^{2} \widetilde{\Psi}=\Psi^{2}$.

Next, to prove (2.15), if $w \in A_{p}$ and if $g \in L^{p^{\prime}}\left(w^{-p^{\prime} / p}\right)$, we have

$$
\left|\sum_{j} \int Q_{j} f_{j} g\right|=\left|\sum_{j} \int f_{j} Q_{j} g\right| \leq C_{p^{\prime}, w^{-p^{\prime}} / p}\left\|\left\{\sum_{j}\left|f_{j}\right|^{2}\right\}^{1 / 2}\right\|_{p, w}\|g\|_{p^{\prime}, w^{-p^{\prime} / p}}
$$

where we have used (2.14) for $g$. Using duality for finite sums and a limiting argument, this gives us the inequality

$$
\left\|\sum_{j} Q_{j} f_{j}\right\|_{p, w} \leq C_{p^{\prime}, w^{-p^{\prime}} / p}\left\|\left\{\sum_{j}\left|f_{j}\right|^{2}\right\}^{1 / 2}\right\|_{p, w},
$$

which is one of the claimed variants of (2.15), and if we replace $Q_{j}$ with $\widetilde{Q}_{j}$ (defined in the preceding argument) and $f_{j}$ with $Q_{j} f_{j}$, the observation that $\widetilde{Q}_{j} Q_{j}=Q_{j}$ allows us to conclude (2.15). If we replace $Q_{j}$ with $Q_{j}^{2}$, a similar argument using instead the variant of (2.14) gives the remaining variants of (2.15). Note also that the one of these variants of $(2.15)$ which has $Q_{j}^{2}$ in place of $Q_{j}$ on both sides follows immediately by applying the original inequality to the sequence $\left\{Q_{j} f_{j}\right\}$.

We turn now to some of the senses in which the identity $\sum_{j} Q_{j}^{2} f=f$ holds. We first see that it holds in the pointwise sense, i.e.,

$$
\lim _{k, l \rightarrow \infty} \sum_{j=-k}^{l} Q_{j}^{2} f(x)=f(x)
$$

for all $x$ if $f$ is a continuous function which vanishes at infinity. To see this, let $\varphi$ be the Schwartz function with integral 1 which is given by $\widehat{\varphi}(\xi)=1-$ $\sum_{j<0} \Psi\left(2^{j}|\xi|\right)^{2}$, and observe that also $\widehat{\varphi}(\xi)=\sum_{j=0}^{\infty} \Psi\left(2^{j}|\xi|\right)^{2}$ for $\xi \neq 0$. Define $\varphi_{j}(x)=2^{-d j} \varphi\left(2^{-j} x\right)$. Then for any distribution $f$,

$$
\sum_{j=-k}^{l} Q_{j}^{2} f=\varphi_{-k} * f-\varphi_{l+1} * f .
$$

When $f$ is a continuous function vanishing at infinity, $\varphi_{-k} * f \rightarrow f$ and $\varphi_{l+1} * f \rightarrow 0$ pointwise everywhere as $k, l \rightarrow \infty$, and (2.20) follows. In particular, (2.20) holds almost everywhere pointwise if $f, \widehat{f} \in L^{1}\left(\mathbf{R}^{d}\right)$, because then $f$ can be redefined on a set of measure 0 so that it is a continuous function vanishing at infinity. Using (2.21) and differentiation theory we can also see that (2.20) holds pointwise a.e. and in $L^{p}(w)$ norm for any $f \in L^{p}(w)$ if $w \in A_{p}, 1<p<\infty$. Although we will not use the fact, $(2.20)$ can be shown to hold in the sense of distributions (i.e., in $\mathcal{S}^{\prime}$ ) for any distribution $f$ such that $\left\|\varphi_{n} * f\right\|_{\infty} \rightarrow 0$ as $n \rightarrow \infty$.

As the Littlewood-Paley inequalities deal with weighted norms of $\ell^{2}$-valued terms, we shall also need vector-valued inequalities for the Hardy-Littlewood maximal operator $M$. In [AJ], K. F. Andersen and R. T. John prove that if $1<p, q<\infty$ 
and if $w \in A_{p}$, then

$$
\left\|\left\{\sum_{j}\left|M f_{j}\right|^{q}\right\}^{1 / q}\right\|_{p, w} \leq C_{p, q, w}\left\|\left\{\sum_{j}\left|f_{j}\right|^{q}\right\}^{1 / q}\right\|_{p, w},
$$

where the constant may be taken to depend only on the $A_{p}$-constant of $w$. (This is a generalization to the weighted setting of the unweighted inequalities first proven by C. Fefferman and E. M. Stein in [FS].)

In order to use the preceding facts in a proof of Theorem 1.2, we shall need to define some intermediate operators which we will use in describing the integral representation (1.2) of $\mathrm{T}_{\Omega}$ and the auxiliary functions $G_{j}$. For $s \in \mathbf{Z}, m \geq 0$, let

$$
B_{s} f(x)=\int_{2^{s-1}}^{2^{s}} A_{t} f(x) \frac{d t}{t}, \quad \text { and } \quad B_{s}^{m} f(x)=\int_{2^{s-1}}^{2^{s}} A_{t}^{m} f(x) \frac{d t}{t} .
$$

Note that the operators $A_{t}, A_{t}^{m}, B_{s}, B_{s}^{m}$ are given by convolution with functions $a_{t}(\cdot), a_{t}^{m}(\cdot), b_{s}(\cdot), b_{s}^{m}(\cdot)$, respectively, defined by

$$
\begin{aligned}
a_{t}(x) & =\frac{1}{t^{d}} \chi_{t S}(x) \operatorname{sgn} \Omega(x), & a_{t}^{m}(x) & =\frac{1}{t^{d}} \chi_{t S_{m}}(x) \operatorname{sgn} \Omega(x), \\
b_{s}(x) & =\int_{2^{s-1}}^{2^{s}} a_{t}(x) \frac{d t}{t}, & b_{s}^{m}(x) & =\int_{2^{s-1}}^{2^{s}} a_{t}^{m}(x) \frac{d t}{t} .
\end{aligned}
$$

This being so, we also have the following integral bounds:

$$
\begin{aligned}
& \int\left|a_{t}^{m}(x)\right| d x \leq\left|S_{m}\right|, \\
& \int a_{t}^{m}(x) d x=\int_{S_{m}} \operatorname{sgn} \Omega(x) d x=\frac{1}{d} \int_{\Theta_{m}} \Omega(\theta) d \theta, \\
& \int\left|b_{s}^{m}(x)\right| d x \leq(\ln 2)\left|S_{m}\right| \leq\left|S_{m}\right|, \\
& \int b_{s}^{m}(x) d x=\ln 2 \int_{S_{m}} \operatorname{sgn} \Omega(x) d x=\frac{\ln 2}{d} \int_{\Theta_{m}} \Omega(\theta) d \theta .
\end{aligned}
$$

We have $B_{s} f=\sum_{m \geq 0} B_{s}^{m} f$, and

$$
\int_{0}^{\infty} A_{t} f \frac{d t}{t}=\sum_{s \in \mathbf{Z}} B_{s} f=\sum_{s \in \mathbf{Z}} \sum_{m \geq 0} B_{s}^{m} f
$$

For this last representation, there is no problem writing the sums in the given order if $f, \widehat{f} \in L^{1}\left(\mathbf{R}^{d}\right)$, as the inner sum converges absolutely to $B_{s} f$ (since $\left\|b_{s}^{m}\right\|_{1} \leq(\ln 2)\left|S_{m}\right|$ and $\left.\sum\left|S_{m}\right|=|S|<\infty\right)$, and $\sum_{s} B_{s} f$ converges absolutely, this being implied by the absolute convergence of $\int_{0}^{\infty} A_{t} f(x) d t / t$.

We also see that we can rewrite (1.3) as

$$
G_{j} f=\sum_{m=0}^{\infty} \sum_{s=0}^{N m} B_{s+j-m}^{m} f,
$$

where, recall, $N$ is a large integer independent of $j$ which may vary with use. Note that $G_{j}$ is given by convolution with a function which is equal to the corresponding sum of the functions $b_{s+j-m}^{m}$, and so has $L^{1}$ norm bounded by $\sum_{m \geq 0}(1+N m)\left|S_{m}\right| \approx$ 
$\int_{d-1}|\Omega(\theta)|\left(1+N \log ^{+}|\Omega(\theta)|\right) d \theta$. Thus the sum for $G_{j}$ converges absolutely if $f$ is bounded, and converges absolutely almost everywhere if $f \in L^{p}, 1 \leq p<\infty$.

We wish to first prove Theorem 1.2 under the assumption that $\Omega$ satisfies the extra cancellation condition

$$
\int_{\Theta_{m}} \Omega(\theta) d \theta=0 \quad \text { for all } m \geq 0 .
$$

This happens for example if $\Omega$ is odd, and we will see later how to prove Theorem 1.2 without this condition. By (2.24) and (2.26), condition (2.29) means that

$$
\int a_{t}^{m}(x) d x=0 \quad \text { and } \quad \int b_{s}^{m}(x) d x=0
$$

for all $m, t$ and $s$, so that $A_{t}^{m}$ and $B_{s}^{m}$ annihilate constants just as $A_{t}$ and $B_{s}$ do. We will use this cancellation to justify the following decomposition.

Lemma 2.1. If $\Omega \in L \log L\left(\mathbf{S}^{d-1}\right)$ satisfies the strong cancellation condition (2.29) and if $f, \widehat{f} \in L^{1}\left(\mathbf{R}^{d}\right)$ (in particular if $f \in \mathcal{S}$ ), then

$$
\int_{0}^{\infty} A_{t} f(x) \frac{d t}{t}=\sum_{j \in \mathbf{Z}} \sum_{s \in \mathbf{Z}} \sum_{m \geq 0} Q_{j}^{2} B_{s}^{m} f(x)
$$

where the sum on the right converges absolutely (and so is independent of rearrangement) for all $x$ to a bounded function.

Proof of Lemma 2.1. We will show that

$$
\sum_{m \geq 0} \sum_{s \in \mathbf{Z}}\left|\widehat{b}_{s}^{m}(\xi)\right| \leq C_{\Omega}
$$

for all $\xi \neq 0$, which by the properties of $\Psi$ tells us that

$$
\sum_{j \in \mathbf{Z}} \sum_{s \in \mathbf{Z}} \sum_{m \geq 0}\left|\Psi\left(2^{j}|\xi|\right)\right|^{2}\left|\widehat{b}_{s}^{m}(\xi)\right| \leq C_{\Omega}
$$

for all $\xi \neq 0$. The lemma will then follow. Indeed, the operators $Q_{j}^{2}, B_{s}^{m}$ are given by convolution with integrable functions, so that

$$
Q_{j}^{2} B_{s}^{m} f(x)=\left(\Psi\left(2^{j}|\cdot|\right)^{2} \widehat{b}_{s}^{m} \widehat{f}\right)^{\vee}(x)
$$

pointwise for all $x$ if $f, \widehat{f} \in L^{1}\left(\mathbf{R}^{d}\right)$, in which case taking absolute values, summing and interchanging the order of summation and integration gives

$$
\begin{aligned}
\sum_{j \in \mathbf{Z}} \sum_{s \in \mathbf{Z}} \sum_{m \geq 0}\left|Q_{j}^{2} B_{s}^{m} f(x)\right| & \leq \int_{\mathbf{R}^{d}}|\widehat{f}(\xi)| \sum_{j \in \mathbf{Z}} \sum_{s \in \mathbf{Z}} \sum_{m \geq 0}\left|\Psi\left(2^{j}|\xi|\right)\right|^{2} \widehat{b}_{s}^{m}(\xi) \mid \\
& \leq C_{\Omega}\|\widehat{f}\|_{1} .
\end{aligned}
$$

On the other hand, (2.31) also implies that

$$
\sum_{s \in \mathbf{Z}} \sum_{m \geq 0}\left|B_{s}^{m} f(x)\right| \leq \int_{\mathbf{R}^{d}}|\widehat{f}(\xi)| \sum_{s \in \mathbf{Z}} \sum_{m \geq 0}\left|\widehat{b}_{s}^{m}(\xi)\right| d \xi \leq C_{\Omega}\|\widehat{f}\|_{1}
$$

for all $x$, and consequently, by the Lebesgue dominated convergence theorem, for each $j$ and all $x$,

$$
Q_{j}^{2} \sum_{s \in \mathbf{Z}} \sum_{m \geq 0} B_{s}^{m} f(x)=\sum_{s \in \mathbf{Z}} \sum_{m \geq 0} Q_{j}^{2} B_{s}^{m} f(x),
$$


with all sums converging absolutely. The expression on the left here equals

$$
Q_{j}^{2} \int_{0}^{\infty} A_{t} f(x) \frac{d t}{t}
$$

by (2.27), and (2.30) now follows by summing over $j$ and applying (2.20) to the function $\int_{0}^{\infty} A_{t} f(x) d t / t$, which is continuous and vanishes at $\infty$ due to (2.2), (2.4) and the formula preceding (2.4) (recall that $\widehat{f} \in L^{1}$ by assumption).

We will therefore be done once we show (2.31), which we prove from the following estimates:

$$
\begin{gathered}
\left|\widehat{b}_{s}^{m}(\xi)\right| \leq(\ln 2)\left|S_{m}\right|\left|2^{m+s} \xi\right|, \\
\left|\widehat{b}_{s}^{m}(\xi)\right| \leq(\ln 2)\left|S_{m}\right|, \\
\left|\widehat{b}_{s}^{m}(\xi)\right| \leq C_{\alpha, d} 2^{d m}\left|2^{m+s} \xi\right|^{-\alpha}, \quad 0<\alpha<1 .
\end{gathered}
$$

We will use these estimates again in the proof of Theorem 1.2. We get (2.33) directly from (2.25), and to prove (2.32) we again use (2.25) together with the fact that $b_{s}^{m}$ has integral 0 (since we are assuming (2.29)) and is supported in $|x| \leq 2^{s+m}$. We prove (2.34) using an argument similar to one appearing in [DR]. By interpolating between the two bounds appearing in (2.10), we see that the integral which appears there is also bounded by $c_{d}|\Omega(\theta)|(t v(\theta))^{-\alpha}=c_{d} \rho(\theta)^{d-\alpha}|t \xi \cdot \theta|^{-\alpha}$ for $0<\alpha<1$. The function $|\xi \cdot \theta|^{-\alpha}$ is an integrable singularity in $\theta$, and since $\rho(\theta) \leq 2^{m}$ for $\theta \in \Theta_{m}$, we have

$$
\begin{aligned}
\left|\widehat{a}_{t}^{m}(\xi)\right| & =\left|\int_{\Theta_{m}}(\operatorname{sgn} \Omega(\theta)) \int_{0}^{\rho(\theta)} e^{-2 \pi i t r \theta \cdot \xi} r^{d-1} d r d \theta\right| \\
& \leq c_{d} \int_{\mathbf{S}^{d-1}} 2^{d m} \frac{1}{\left|2^{m} t \xi \cdot \theta\right|^{\alpha}} d \theta \leq C_{\alpha, d} \frac{2^{d m}}{\left|2^{m} t \xi\right|^{\alpha}}
\end{aligned}
$$

giving (2.34).

Given $\xi \neq 0$, let $D$ be the integer such that $1 / 2 \leq\left|2^{D} \xi\right|<1$, and for an integer $N>0$ yet to be determined, write

$$
\begin{aligned}
\sum_{m \geq 0} \sum_{s \in \mathbf{Z}}\left|\widehat{b}_{s}^{m}(\xi)\right| & =\sum_{m \geq 0} \sum_{s \in \mathbf{Z}}\left|\widehat{b}_{s+D-m}^{m}(\xi)\right| \\
& =\sum_{m \geq 0} \sum_{s<0}+\sum_{m \geq 0} \sum_{s=0}^{N m}+\sum_{m \geq 0} \sum_{s=N m+1}^{\infty} \\
& =I+I I+I I I .
\end{aligned}
$$

The terms $I, I I, I I I$ strongly parallel terms with the same names which will appear in the Littlewood-Paley argument. To bound these sums, use (2.32) to see that

$$
I \leq \sum_{m \geq 0} \sum_{s=-\infty}^{-1}\left|S_{m}\right|\left|2^{D+s} \xi\right| \leq|S|,
$$


and use (2.33) to see that

$$
I I \leq \sum_{m \geq 0}(N m+1)\left|S_{m}\right| \leq c N\|\Omega\|_{L \log L},
$$

which is a bound depending only on our ultimate choice of $N$. Fixing $\alpha$ with $0<\alpha<1$, we now choose $N$ large enough so that $N \alpha>d$, and using (2.34) we have

$$
I I I \leq C_{\alpha} \sum_{m \geq 0} \sum_{s=N m+1}^{\infty} 2^{d m}\left|2^{D+s} \xi\right|^{-\alpha} \leq C_{\alpha}^{\prime} \sum_{m \geq 0} 2^{d m} 2^{-\alpha N m}=C_{N, \alpha},
$$

for $C_{N, \alpha}$ independent of $\xi$. We have thus established (2.31) and finished the proof of Lemma 2.1.

Now that we have established (2.30) for a class of functions including $\mathcal{S}$ and have shown that the sum is independent of the order of summation, we are justified in writing for such $f$

$$
\begin{aligned}
\int_{0}^{\infty} A_{t} f(x) \frac{d t}{t} & =\sum_{j \in \mathbf{Z}} \sum_{s \in \mathbf{Z}} \sum_{m \geq 0} Q_{j}^{2} B_{s}^{m} f(x) \\
& =\sum_{j \in \mathbf{Z}} \sum_{m \geq 0} \sum_{s \in \mathbf{Z}} Q_{j}^{2} B_{s+j-m}^{m} f(x) \\
& =\sum_{j \in \mathbf{Z}} \sum_{\substack{m \geq 0, s<0}}+\sum_{j \in \mathbf{Z}} \sum_{\substack{m \geq 0, 0 \leq s \leq N m}}+\sum_{j \in \mathbf{Z}} \sum_{\substack{m>0 \\
s>N m}} \\
& =I+I I+I I I,
\end{aligned}
$$

and we need to show appropriate norm inequalities for the terms $I, I I, I I I$. Observe the similarities between the terms $I, I I, I I I$ appearing here and those appearing in the proof of Lemma 2.1.

We begin with $I I$. Note that

$$
I I=\sum_{j} Q_{j}^{2} G_{j} f
$$

and observe by one of the described variants of (2.15) that, since $w \in A_{p}$,

$$
\|I I\|_{p, w} \leq C_{p, w}\left\|\left\{\sum_{j}\left|G_{j} Q_{j}^{2} f\right|^{2}\right\}^{1 / 2}\right\|_{p, w}
$$

( $Q_{j}$ and $G_{j}$ commute with each other, as each is given by a convolution.) Applying (1.4) to the right side of this inequality, we obtain

$$
\|I I\|_{p, w} \leq C_{p, w}^{\prime}\left\|\left\{\sum_{j}\left|Q_{j}^{2} f\right|^{2}\right\}^{1 / 2}\right\|_{p, w} \leq C_{p, w}^{\prime \prime}\|f\|_{p, w},
$$

the last inequality holding by the variant of (2.14) mentioned earlier.

The estimations of $I$ and $I I I$ also begin with the use of (2.15) and end with the use of (2.14). They only vary in the means used to estimate the inequality in the middle. In order to estimate $I I I$, we will need (2.34). This together with the support of the function $\Psi$ used to define $Q_{j}$ yields

$$
\left|\left(Q_{j} B_{s+j-m}^{m} f\right)^{\curlyvee}(\xi)\right| \leq C 2^{d m} 2^{-\alpha s}|\widehat{f}(\xi)| \chi\left\{2^{-j-1}<|\xi|<2^{-j+1}\right\} .
$$


Consequently, by Plancherel's theorem, for any sequence $\left\{f_{j}\right\}$,

$$
\left\|\left\{\sum_{j}\left|Q_{j} B_{s+j-m}^{m} f_{j}\right|^{2}\right\}^{1 / 2}\right\|_{2} \leq C 2^{d m} 2^{-\alpha s}\left\|\left\{\sum_{j}\left|f_{j}\right|^{2}\right\}^{1 / 2}\right\|_{2} .
$$

This $L^{2}$ bound will be used with some pointwise estimates to give weighted norm inequalities. We can crudely estimate $\left|B_{s+j-m}^{m} f(x)\right| \leq C 2^{d m} M f(x)$, since $B_{s+j-m}^{m} f(x)$ is given by convolution with a function supported in a ball of radius $2^{s+j}$ and bounded by $2^{d(m-s-j)}$. We similarly obtain $\left|Q_{j} f(x)\right| \leq C M f(x)$ since $Q_{j}$ can be written as convolution with a Schwartz function $\psi_{j}(x)$ satisfying (2.19). Putting these together gives

$$
\left|Q_{j} B_{s+j-m}^{m} f(x)\right| \leq C 2^{d m} M M f(x) .
$$

Thus, using (2.22) twice, we obtain the crude weighted inequality

$$
\left\|\left\{\sum_{j}\left|Q_{j} B_{s+j-m}^{m} f_{j}\right|^{2}\right\}^{1 / 2}\right\|_{p, w} \leq C_{p, w} 2^{d m}\left\|\left\{\sum_{j}\left|f_{j}\right|^{2}\right\}^{1 / 2}\right\|_{p, w},
$$

for $1<p<\infty$ and $w \in A_{p}$. To use this, we will need to use a result of E. M. Stein and G. Weiss on interpolation with change of measures. (We state their result in a restricted form for our convenience.)

Theorem [SW1]. For $p_{0}, p_{1} \geq 1$ suppose that $T$ is a linear operator satisfying

$$
\|T f\|_{p_{i}, u_{i}} \leq K_{i}\|f\|_{p_{i}, v_{i}}
$$

for all $f$ in $L^{p_{i}}\left(v_{i}\right), i=0,1$. For $0 \leq t \leq 1$, let $p_{t}$ be given by $1 / p_{t}=(1-t) / p_{0}+t / p_{1}$ and let $0 \leq r=r(t) \leq 1$ be defined by $r=t p_{t} / p_{1}$. For such $t$ and associated $r$, define weights $u_{t}=u_{0}^{1-r} u_{1}^{r}$ and $v_{t}=v_{0}^{1-r} v_{1}^{r}$. Then

$$
\|T f\|_{p_{t}, u_{t}} \leq K_{0}^{1-t} K_{1}^{t}\|f\|_{p_{t}, v_{t}}
$$

for all $f \in L^{p_{t}}\left(v_{t}\right)$. (We note that in the case $p_{0}=p_{1}$ this gives $p_{t}=p_{0}$ and $r=t$.)

The general result of [SW1] deals with the case that $T$ is bounded from $L^{s_{i}}\left(v_{i}\right)$ into $L^{p_{i}}\left(u_{i}\right)$. We will only need the case $p_{i}=s_{i}$ that we have stated. The proof in [SW1] extends easily to Banach space-valued functions, and one can in fact show the following result, which we shall need: For $1 \leq p, q<\infty$ and a weight $w$, let

$$
L^{p}\left(\ell^{q} ; w\right)=\left\{\left\{f_{j}\right\}:\left\|\left\{f_{j}\right\}\right\|_{L^{p}\left(\ell^{q}, w\right)}=\left\|\left\{\sum_{j}\left|f_{j}^{q}\right|\right\}^{1 / q}\right\|_{p, w}<\infty\right\},
$$

with the obvious modifications if $p=\infty$ or $q=\infty$. If $1 \leq p_{i}<\infty$ and $1 \leq q_{i} \leq \infty$ and $T$ is a linear operator which is bounded from $L^{p_{i}}\left(\ell^{q_{i}} ; v_{i}\right)$ to $L^{p_{i}}\left(\ell^{q_{i}} ; u_{i}\right)$ with operator norm $\leq K_{i}$ for $i=0,1$, then $T$ is also bounded from $L^{p_{t}}\left(\ell^{q_{t}} ; v_{t}\right)$ to $L^{p_{t}}\left(\ell^{q_{t}} ; u_{t}\right)$ with norm $\leq K_{0}^{1-t} K_{1}^{t}$ for $0<t<1$, where $p_{t}, u_{t}$ and $v_{t}$ are as defined above and $q_{t}$ is defined analogously to $p_{t}$. A straightforward modification of the proof of [SW1] yields this extension, which we shall use in $\S 5$. For related extensions, see $[\mathrm{T}]$.

We may view (2.39) and (2.38) as norm inequalities for a linear operator which takes its values in $\ell^{2}$, and interpolating between (2.39) at some $p_{0}>1$ and (2.38), we see that if $p$ is between $p_{0}$ and 2 and $\delta, 0<\delta<1$, is given by $\delta=(p-2) /\left(p_{0}-2\right)$, 
then whenever $w \in A_{p_{0}}$,

$$
\left\|\left\{\sum_{j}\left|Q_{j} B_{s+j-m}^{m} f_{j}\right|^{2}\right\}^{1 / 2}\right\|_{p, w^{\delta}} \leq C_{p_{0}, p, w} 2^{d m} 2^{-(1-\delta) 2 \alpha s / p}\left\|\left\{\sum_{j}\left|f_{j}\right|^{2}\right\}^{1 / 2}\right\|_{p, w^{\delta}}
$$

But it is a characteristic of $A_{p}$ weights for $p>1$ that when $w \in A_{p}$ there are $\epsilon, \epsilon^{\prime}>0$ such that $w^{1+\epsilon^{\prime}} \in A_{p-\epsilon}$. Therefore choosing $p_{0}$ sufficiently close to $p$ ensures that $w^{1 / \delta} \in A_{p_{0}}$, so the above inequality with $w^{1 / \delta}$ replacing $w$ gives

$$
\left\|\left\{\sum_{j}\left|Q_{j} B_{s+j-m}^{m} f_{j}\right|^{2}\right\}^{1 / 2}\right\|_{p, w} \leq C_{p, w} 2^{d m} 2^{-\eta s}\left\|\left\{\sum_{j}\left|f_{j}\right|^{2}\right\}^{1 / 2}\right\|_{p, w},
$$

for some $\eta>0$, whenever $1<p<\infty$ and $w \in A_{p}$. We can in fact choose $C_{p, w}$ and $\eta$ depending only on $p$ and the $A_{p}$ constant of $w$.

We are now ready to estimate $I I I$. Using (2.15) and the triangle inequality, we see that

$$
\begin{aligned}
\|I I I\|_{p, w} & \leq\left\|\left\{\sum_{j}\left|\sum_{\substack{m>0 \\
s>N m}} Q_{j} B_{s+j-m}^{m} Q_{j} f\right|^{2}\right\}^{1 / 2}\right\|_{p, w} \\
& \leq \sum_{\substack{m>0 \\
s>N m}}\left\|\left\{\sum_{j}\left|Q_{j} B_{s+j-m}^{m} Q_{j} f\right|^{2}\right\}^{1 / 2}\right\|_{p, w},
\end{aligned}
$$

so if we choose $N$ so that $N \eta>d$, applying (2.4) to the right side of the above inequality gives the bound

$$
\begin{aligned}
\|I I I\|_{p, w} & \leq C_{p, w} \sum_{\substack{m \geq 0 \\
s>N m}} 2^{d m} 2^{-\eta s}\left\|\left\{\sum_{j}\left|Q_{j} f\right|^{2}\right\}^{1 / 2}\right\|_{p, w} \\
& \leq C_{p, w}^{\prime} \sum_{m \geq 0} 2^{(d-N \eta) m}\left\|\left\{\sum_{j}\left|Q_{j} f\right|^{2}\right\}^{1 / 2}\right\|_{p, w} \\
& \leq C_{p, w}^{\prime \prime}\|f\|_{p, w} .
\end{aligned}
$$

We now derive a similar estimate for $I$. Since $b_{s}^{m}$ is supported in $|x|<2^{s+m}$, has integral 0 and $L^{1}$ norm at most $(\ln 2)\left|S_{m}\right|($ by $(2.29),(2.26)$ and $(2.25)$ ), we see that $\sum_{m \geq 0} B_{s-m}^{m}$ is given by convolution with a function $k_{s}$ supported in $|x|<2^{s}$, with integral 0 and $L^{1}$ norm at most $(\ln 2)|S|$. We again use the fact that $Q_{j}$ is given by convolution with a Schwartz function $\psi_{j}(x)$ satisfying (2.19). At $j=0$, this tells us that

$$
\left|\psi_{0}(x-y)-\psi_{0}(x)\right| \leq \frac{C|y|}{(1+|x|)^{d+1}} \quad \text { for all }|y| \leq 1 \text { and all } x \in \mathbf{R}^{d} .
$$

Using this for $s<0$ together with the facts about the support and cancellation of $k_{s}$, we see that $\psi_{0} * k_{s}$, the convolution kernel of the operator $Q_{0}\left(\sum_{m \geq 0} B_{s-m}^{m}\right)$, satisfies

$$
\begin{aligned}
\left|\psi_{0} * k_{s}(x)\right| & =\left|\int_{|y|<2^{s}} k_{s}(y)\left[\psi_{0}(x-y)-\psi_{0}(x)\right] d y\right| \\
& \leq \frac{C 2^{s}}{(1+|x|)^{d+1}} .
\end{aligned}
$$


In this inequality, $C=C^{\prime}\left\|k_{s}\right\|_{1} \leq C^{\prime \prime}|S|$ for $C^{\prime \prime}$ independent of $\Omega$. Now, all of the functions $\psi_{j}(x), b_{j}$, and $k_{j}$ scale with $j$ by dilation, i.e., $\psi_{j}(x)=2^{-d j} \psi_{0}\left(2^{-j} x\right)$, and similarly for $b_{j}^{m}$, etc. Consequently, we have $\psi_{j} * k_{j+s}(x)=2^{-d j} \psi_{0} * k_{s}\left(2^{-j} x\right)$, and summing the above inequality for $s<0$ and rescaling, we see that $Q_{j}\left(\sum_{\substack{m>0 \\ s<0}} B_{s+j-m}^{m}\right)$ is given by convolution with a function bounded by $C 2^{-d j} /\left(1+\left|2^{-j} x\right|\right)^{d+1}$ uniformly in $j \in \mathbf{Z}$, and therefore

$$
\left|Q_{j}\left(\sum_{\substack{m>0 \\ s<0}} B_{s+j-m}^{m}\right) f(x)\right| \leq C M f(x)
$$

with similar uniformity. We may now use this with (2.22) to get the bound for $I$ in essentially the way we estimated $I I I$. Specifically,

$$
\begin{aligned}
\|I\|_{p, w} & \leq C_{1}\left\|\left\{\sum_{j}\left|\sum_{\substack{m>0 \\
s<0}} Q_{j} B_{j+s-m}^{m} Q_{j} f\right|^{2}\right\}^{1 / 2}\right\|_{p, w} \\
& \leq C_{2}\left\|\left\{\sum_{j}\left|M\left(Q_{j} f\right)\right|^{2}\right\}^{1 / 2}\right\| \|_{p, w} \\
& \leq C_{3}\left\|\left\{\sum_{j}\left|Q_{j} f\right|^{2}\right\}^{1 / 2}\right\|_{p, w} \\
& \leq C_{4}\|f\|_{p, w} .
\end{aligned}
$$

Here, the first and last lines use our Littlewood-Paley inequalities, the second line uses the inequality we just derived, and the following line uses (2.22).

This completes the proof of Theorem 1.2 assuming the extra cancellation (2.29). To eliminate this assumption, first define a modified version $\widetilde{A}_{t}^{m}$ of $A_{t}^{m}$ by

$$
\begin{aligned}
\widetilde{A}_{t}^{m} f(x) & =A_{t}^{m} f(x)-\frac{c_{m}}{\left|t B_{1}(0)\right|} \int_{t B_{1}(0)} f(x-y) d y \\
& =\int_{\mathbf{R}^{d}} f(x-y) \widetilde{a}_{t}^{m}(y) d y
\end{aligned}
$$

where $B_{1}(0)$ is the unit ball in $\mathbf{R}^{d}$ centered at the origin and

$$
c_{m}=\int_{\mathbf{R}^{d}} a_{t}^{m}(y) d y .
$$

Here $\widetilde{a}_{t}^{m}$ is given by

$$
\begin{aligned}
\widetilde{a}_{t}^{m}(y) & =a_{t}^{m}(y)-\frac{c_{m}}{\left|t B_{1}(0)\right|} \chi_{t B_{1}(0)}(y) \\
& =\frac{1}{t^{d}}\left[\chi_{t S_{m}}(y) \operatorname{sgn} \Omega(y)-\frac{c_{m}}{\left|B_{1}(0)\right|} \chi_{t B_{1}(0)}(y)\right] .
\end{aligned}
$$

Note from the definition of $c_{m}$ and by (2.23) that

$$
\int_{\mathbf{R}^{d}} \widetilde{a}_{t}^{m}(y) d y=0, \quad \text { and } \quad \int_{\mathbf{R}^{d}}\left|\widetilde{a}_{t}^{m}(y)\right| d y \leq 2 \int_{\mathbf{R}^{d}}\left|a_{t}^{m}(y)\right| d y \leq 2\left|S_{m}\right|,
$$


and also that $\sum_{m \geq 0}\left|c_{m}\right| \leq|S|$. In particular this says that $\widetilde{A}_{t}^{m}$ annihilates constants.

Now define $\widetilde{B}_{s}^{m}$ from $\widetilde{A}_{t}^{m}$ in the same way $B_{s}^{m}$ was defined from $A_{t}^{m}$, by letting

$$
\widetilde{B}_{s}^{m}=\int_{2^{s-1}}^{2^{s}} \widetilde{A}_{t}^{m} \frac{d t}{t}
$$

Note also that $B_{s}^{m} f=f * \widetilde{b}_{s}^{m}$, where

$$
\widetilde{b}_{s}^{m}(y)=\int_{2^{s-1}}^{2^{s}} \widetilde{a}_{t}^{m}(y) \frac{d t}{t} .
$$

Since $\Omega$ has integral 0 , then $\sum_{m \geq 0} c_{m}=0$ (see, e.g. (2.24)), so we have

$$
A_{t}=\sum_{m \geq 0} \widetilde{A}_{t}^{m}, \quad B_{s}=\sum_{m \geq 0} \widetilde{B}_{s}^{m}
$$

and

$$
\int_{0}^{\infty} A_{t} f(x) \frac{d t}{t}=\sum_{s \in \mathbf{Z}} B_{s} f(x)=\sum_{s \in \mathbf{Z}} \sum_{m \geq 0} \widetilde{B}_{s}^{m} f(x) .
$$

We now claim that the proof of Theorem 1.2 which we gave under the cancellation requirement (2.29) holds in the general case if we replace $A_{t}^{m}, B_{s}^{m}$ with $\widetilde{A}_{t}^{m}, \widetilde{B}_{s}^{m}$. Specifically, to see that the analogue of Lemma 2.1 holds, observe that $\widetilde{b}_{s}^{m}(x)$ is supported in $|x| \leq 2^{m+s}$ and

$$
\begin{aligned}
& \left|\left(\widetilde{b}_{s}^{m}\right) \hat{(\xi)}\right| \leq 2(\ln 2)\left|S_{m}\right|\left|2^{m+s} \xi\right|, \\
& \left|\left(\widetilde{b}_{s}^{m}\right) \hat{(\xi)}\right| \leq 2(\ln 2)\left|S_{m}\right|, \\
& \left|\left(\widetilde{b}_{s}^{m}\right) \hat{(\xi)}\right| \leq C_{\alpha} 2^{d m}\left|2^{m+s} \xi\right|^{-\alpha}, \quad 0<\alpha<1 .
\end{aligned}
$$

These may be obtained by integrating in $t$ similar inequalities for $\left(\widetilde{a}_{t}^{m}\right)^{\wedge}$, namely, $\left|\left(\widetilde{a}_{t}^{m}\right) \hat{(\xi)}\right|$ has the three bounds $2\left|S_{m}\right|\left|2^{m} t \xi\right|, 2\left|S_{m}\right|$ and $C_{\alpha, d} 2^{d m}\left|2^{m} t \xi\right|^{-\alpha}$ for $0<$ $\alpha<1$. The first two of these bounds can be obtained by reasoning as for (2.32) and (2.33), and the last one follows from the estimate for $\widehat{a}_{t}^{m}$ given after (2.34), using also the estimate $\left|\left(\chi_{t B_{1}(0)}\right) \hat{(\xi)}\right| \leq c t^{d}|t \xi|^{-d / 2}$ (see, e.g., [SW2, p. 171]; the parameter $\delta$ there may also be 0 , as the proof shows).

Comparing these to $(2.32)-(2.34)$, we see that $\widetilde{B}_{s}^{m}$ effectively satisfies the same inequalities for general $\Omega \in L \log L\left(\mathbf{S}^{d-1}\right)$ with integral 0 (as in Theorem 1.2) that $B_{s}^{m}$ satisfies under the stronger cancellation condition (2.29).

If now $I, I I$ and $I I I$ are the corresponding terms with $\widetilde{B}_{j}^{m}$ replacing $B_{j}^{m}$, then the estimation of the terms $I$ and $I I I$ proceeds roughly as before because the modification leaves essentially unchanged the size, support and Fourier transform bounds which were used to estimate these terms.

For the term $I I$, since $\widetilde{A}_{t}^{m}$ differs from $A_{t}^{m}$ by $c_{m}$ times an average over a ball of radius $t$, then we see that the difference between the original definition of $G_{j} f(x)$ and the corresponding sum of the modified terms $\widetilde{B}_{s}^{m} f(x)$ can be bounded by a constant times $M f(x)$, the constant being a geometric multiple of

$$
\sum_{m \geq 0}(1+N m)\left|c_{m}\right|
$$


But this constant is finite because we know $c_{m} \leq\left|S_{m}\right|$ and $\Omega \in L \log L\left(\mathbf{S}^{d-1}\right)$. Since $w \in A_{p}$, we have from (2.22) the square inequalities

$$
\left\|\left\{\sum_{j}\left|M f_{j}\right|^{2}\right\}^{1 / 2}\right\|_{p, w} \leq C_{p, w}\left\|\left\{\sum_{j}\left|f_{j}\right|^{2}\right\}^{1 / 2}\right\|_{p, w} .
$$

From these two considerations, we see that the square inequality (1.4) for the original terms $G_{j}$ implies up to a fixed factor the same inequality for the modified terms, which then yields the desired estimate for $I I$. This completes the proof of Theorem 1.2 .

\section{EXAMPLES}

Having proven Theorems 1.1 and 1.2, we give an example showing that the conditions (1.7), (1.9) and (1.10) of Theorems 1.4 and 1.5 are fairly sharp before going on to prove them. Observe that, for $1<p<\infty$, the following inequality is implied by any of the weight conditions (1.7), (1.9) or (1.10):

$$
\left(\frac{1}{|R|} \int_{R} w(x) d x\right)^{1 / p}\left(\frac{1}{|R|} \int_{R} w(x)^{-p^{\prime} / p} d x\right)^{1 / p^{\prime}} \leq \frac{c_{m, k}}{\left|R_{m, k}\right|},
$$

for every rectangle $R$ which is a translate and dilate of the rectangle $R_{m, k}$. In fact, for $p=2$ this is the inequality (1.7), and it follows from (1.9) for $1<p \leq 2$, and from (1.10) for $2 \leq p \leq \infty$, by using Hölder's inequality. Since (3.1) is really an $A_{p}$ condition for a different choice of scale, then by applying the "reverse Hölder" argument in $[\mathrm{CF}]$ for each choice of rectangle $R_{m, k}$, we see that if (3.1) holds for some choice of constants $c_{m, k}$, then for each $m, k$, there is some other choice of constants $c_{m, k}$ and a constant $r=r_{m, k}>1$ depending on the original constant in (3.1) so that (1.9) and (1.10) hold in their respective ranges of $p$.

In this section, we give some simple examples showing that the conditions (1.7), (1.9) and (1.10) are fairly sharp for $p$ in the appropriate ranges. We will show that if $\mathrm{T}_{\Omega}$ is bounded on $L^{p}(w)$ for suitable choices of $\Omega$ with $|\Omega|=\rho^{d}$, i.e., if $1<p<\infty$ and

$$
\int_{\mathbf{R}^{d}}\left|\mathrm{~T}_{\Omega} f\right|^{p} w \leq c \int_{\mathbf{R}^{d}}|f|^{p} w
$$

holds for $f \in L^{p}(w)$, then the inequalities (3.1) hold up to a convergence factor, i.e., with constants $c_{m, k}$ replaced with a single constant $c$ independent of $m$ and $k$. That is, we will give examples of $\Omega$ so that if $1<p<\infty$ and $w$ is a weight for which (3.2) holds, then

$$
\left(\frac{1}{|R|} \int_{R} w(x) d x\right)^{1 / p}\left(\frac{1}{|R|} \int_{R} w(x)^{-p^{\prime} / p} d x\right)^{1 / p^{\prime}} \leq \frac{c}{\left|R_{m, k}\right|}
$$

for every rectangle $R$ which is a translate and dilate of the rectangle $R_{m, k}$. In particular, the example we consider will have $\Omega \in L \log L\left(\mathbf{S}^{d-1}\right)$ and unbounded.

For simplicity, we restrict our examples to the case $d=2$, and we let

$$
K(x)=\frac{\Omega\left(x^{\prime}\right)}{|x|^{2}}, \quad x^{\prime}=\frac{x}{|x|}=(\cos \theta, \sin \theta),
$$


where $\Omega\left(x^{\prime}\right)$ is the odd function of $x^{\prime}$ given for $|\theta| \leq \pi / 2$ by

$$
\Omega\left(x^{\prime}\right)=\frac{1}{|\theta|(1+|\log | \theta||)^{2+\epsilon}}
$$

for some $\epsilon>0$. Then $\Omega \in L \log L\left(\mathbf{S}^{1}\right)$ and has integral 0 over $\mathbf{S}^{1}$. We have chosen $\Omega$ so that it does not belong to $L^{q}\left(\mathbf{S}^{1}\right)$ for any $q>1$, but this is not really important for what follows. Similar considerations would apply to odd $\Omega$ defined by $\Omega\left(x^{\prime}\right)=|\theta|^{-\delta}$ for $|\theta| \leq \pi / 2$ for some $\delta$ with $0<\delta<1$.

Note that for our example the set $S=\{x:|K(x)|>1\}$ is symmetric with respect to the coordinate axes and unbounded along the axis $x_{2}=0$ (writing $\left.x=\left(x_{1}, x_{2}\right)\right)$. The part of $S$ which lies in the first and fourth quadrants is given in polar coordinates by

$$
\left\{x=r \theta: 0 \leq r<\left[|\theta|(1+|\log | \theta||)^{2+\epsilon}\right]^{-1 / 2},|\theta| \leq \pi / 2\right\} .
$$

The collection of basic rectangles which cover $S$ is then composed of rectangles $R_{m}, m=1,2, \ldots$, centered at the origin and of the form (up to dilation by a fixed constant multiple)

$$
R_{m}=\left[-2^{m}, 2^{m}\right] \times\left[-\frac{1}{2^{m} m^{2+\epsilon}}, \frac{1}{2^{m} m^{2+\epsilon}}\right]
$$

Given a weight $w$ for which (3.2) is valid when $\mathrm{T}_{\Omega}$ is the singular integral with kernel $K$, we wish to show that there is a constant $c$ so that (3.3) holds for every $R$ which is a translation or dilation of a rectangle which is in our starlike cover. Since in this example our starlike cover is essentially (that is, up to a single dilation) given by $\left\{R_{m}\right\}$, then we wish to show

$$
\left(\int_{R} w d x\right)^{1 / p}\left(\int_{R} w^{-p^{\prime} / p} d x\right)^{1 / p^{\prime}} \leq c \frac{|R|}{\left|R_{m}\right|},
$$

for every $m$ and every rectangle $R$ which is a translate and dilate of $R_{m}$, with $c$ independent of $R$ and $m$.

To do this, fix $m$ and let $R$ be a translation and dilation of $R_{m}$. Because $\mathrm{T}$ commutes with translations, we may without loss of generality assume that $R$ is centered at the origin, so that we can write

$$
R=s R_{m}=\left[-s 2^{m}, s 2^{m}\right] \times\left[-\frac{s}{2^{m} m^{2+\epsilon}}, \frac{s}{2^{m} m^{2+\epsilon}}\right]
$$

for some $s>0$. We may also assume that the second integral in (3.4) is positive and finite. Partition $R$ by a vertical line $x_{1}=\alpha$ into rectangles $R_{-}, R_{+}$so that

$$
\int_{R_{-}} w^{-p^{\prime} / p} d x=\int_{R_{+}} w^{-p^{\prime} / p} d x=\frac{1}{2} \int_{R} w^{-p^{\prime} / p} d x .
$$

Let $R_{-}$be the rectangle which lies to the left of the dividing line and let $R_{+}$be the rectangle which lies to the right.

First pick $f=\chi_{R_{-}} w^{-1 /(p-1)}$ in (3.2) to get

$$
\int_{\mathbf{R}^{d}}\left|\mathrm{~T}_{\Omega}\left(\chi_{R_{-}} w^{-1 /(p-1)}\right)(x)\right|^{p} w(x) d x \leq c \int_{R_{-}} w^{-1 /(p-1)}(x) d x .
$$


Restricting the integration on the left to $R_{+}$and noting that $\Omega(x-y)$ has constant sign when $x \in R_{+}$and $y \in R_{-}$, we obtain

$$
\int_{R_{+}}\left[\int_{R_{-}} w^{-1 /(p-1)}(y) \frac{|\Omega(x-y)|}{|x-y|^{2}} d y\right]^{p} w(x) d x \leq c \int_{R_{-}} w^{-1 /(p-1)}(y) d y .
$$

We claim that

$$
\frac{|\Omega(x-y)|}{|x-y|^{2}} \geq \frac{c}{s^{2}} \quad \text { if } x \in R_{+} \text {and } y \in R_{-},
$$

for some constant $c>0$ independent of $x, y, s$ and $m$. This is a corollary of the homogeneity of the kernel and the definition of $S$, but can also be seen directly as follows. If $x \in R_{+}$and $y \in R_{-}$, then $|x-y| \leq c s 2^{m}$ and the unit vector $(x-y)^{\prime}=(\cos \theta, \sin \theta)$ with $|\theta| \leq \pi / 2$, and we will consider separately the ranges $|\theta|<\left(s / 2^{m} m^{2+\epsilon}\right) / s 2^{m}=\left(2^{2 m} m^{2+\epsilon}\right)^{-1}$ and $\left(2^{2 m} m^{2+\epsilon}\right)^{-1} \leq|\theta| \leq \pi / 2$. These ranges correspond essentially to whether the line through $x$ and $y$ intersects the boundary of $R$ through the vertical or horizontal edges of $R$. In the first case,

$$
|\Omega(x-y)| \geq \frac{c}{|\theta|(|\log | \theta||)^{2+\epsilon}} \geq \frac{c 2^{2 m} m^{2+\epsilon}}{m^{2+\epsilon}}=c 2^{2 m},
$$

so that

$$
\frac{|\Omega(x-y)|}{|x-y|^{2}} \geq c \frac{2^{2 m}}{\left(s 2^{m}\right)^{2}}=\frac{c}{s^{2}} .
$$

In the second case, the maximum value of $|x-y|$ is smaller, satisfying $|x-y| \leq$ $c s /\left(|\theta| 2^{m} m^{2+\epsilon}\right)$, while

$$
|\Omega(x-y)| \geq \frac{c}{|\theta|(1+|\log | \theta||)^{2+\epsilon}} \geq \frac{c}{|\theta| m^{2+\epsilon}} .
$$

Thus, in this case

$$
\begin{aligned}
\frac{|\Omega(x-y)|}{|x-y|^{2}} & \geq \frac{c}{|\theta| m^{2+\epsilon}}\left(\frac{|\theta| 2^{m} m^{2+\epsilon}}{s}\right)^{2} \\
& =\frac{c}{s^{2}}|\theta| 2^{2 m} m^{2+\epsilon} \geq \frac{c}{s^{2}},
\end{aligned}
$$

which proves (3.7) in every case.

Using (3.7) and (3.6) gives

$$
\left(\frac{1}{s^{2}} \int_{R_{-}} w(y)^{-1 /(p-1)} d y\right)^{p}\left(\int_{R_{+}} w(x) d x\right) \leq c\left(\int_{R_{-}} w(y)^{-1 /(p-1)} d y\right) .
$$

Dividing by the integral on the right, using (3.5) and taking the $p$ th root, we obtain

$$
\frac{1}{s^{2}}\left(\frac{1}{2} \int_{R} w(y)^{-1 /(p-1)} d y\right)^{1 / p^{\prime}}\left(\int_{R_{+}} w(x) d x\right)^{1 / p} \leq c,
$$

or since $|R| /\left|R_{m}\right|=s^{2}$,

$$
\left(\int_{R_{+}} w(x) d x\right)^{1 / p}\left(\int_{R} w(y)^{-1 /(p-1)} d y\right)^{1 / p^{\prime}} \leq c \frac{|R|}{\left|R_{m}\right|} .
$$


Next, repeating the entire argument with $R_{-}$and $R_{+}$interchanged (beginning by now choosing $f=\chi_{R_{+}} w^{-1 /(p-1)}$, etc.), we obtain an inequality analogous to $(3.8)$ :

$$
\left(\int_{R_{-}} w(x) d x\right)^{1 / p}\left(\int_{R} w(y)^{-1 /(p-1)} d y\right)^{1 / p^{\prime}} \leq c \frac{|R|}{\left|R_{m}\right|} .
$$

By adding (3.8) and (3.9), we obtain (3.3) and we are done.

\section{Proofs of Theorems 1.3-1.5}

We begin with some general background. Let $S=S(\Omega)$ be the starlike set about the origin associated with the function $\Omega$ which is homogeneous of degree 0 . We first define what we mean by a statified starlike cover of $S$. Recall that $S=\bigcup_{m=0}^{\infty} S_{m}$ where

$$
\begin{aligned}
S_{0} & =\left\{x=r \theta: 0 \leq r \leq \rho(\theta), \theta \in \mathbf{S}^{d-1}, \rho(\theta) \leq 1\right\}, \\
S_{m} & =\left\{x=r \theta: 0 \leq r \leq \rho(\theta), \theta \in \mathbf{S}^{d-1}, 2^{m-1}<\rho(\theta) \leq 2^{m}\right\}, \quad \text { for } m \geq 1,
\end{aligned}
$$

with $\rho(\theta)=|\Omega(\theta)|^{1 / d}$. Using the same sort of construction used in the proof of part (A) of Lemma 2.1 in [CWW], p. 248, we will show that there are rectangles $\left\{R_{m, k}\right\}_{m, k}$ centered at the origin with

$$
S_{m} \subseteq \bigcup_{k} R_{m, k} \quad \text { for } m \geq 0,
$$

the longest edgelength of $R_{m, k} \approx 2^{m}$, and

$$
\sum_{k}\left|R_{m, k}\right| \leq c\left|S_{m}\right| \quad \text { for } m \geq 0
$$

with $c$ depending only on the dimension $d$. Any such covering will be called a stratified starlike cover, the word "stratified " referring to the relation of the rectangles to the subsets $S_{m}$ of $S$. Such a cover always exists, although the following construction is not necessarily optimal. For $m=0$ we pick the single rectangle equal to the cube with center at the origin and edgelength 2 . For $m \geq 1$, cover the set $\Theta_{m}=\left\{\theta \in \mathbf{S}^{d-1}: 2^{m-1}<\rho(\theta) \leq 2^{m}\right\}$ by discs $D_{m, k}=\left\{\theta \in \mathbf{S}^{\bar{d}-1}:\left|\theta-\theta_{m, k}\right|<\right.$ $\left.\epsilon_{m, k}\right\}, \theta_{m, k} \in \mathbf{S}^{d-1}$, satisfying $\sum_{k}\left|D_{m, k}\right| \approx\left|\Theta_{m}\right|$, and to each disc $D_{m, k}$ assign a rectangle $R_{m, k}$ with major axis in the direction $\theta_{m, k}$ so that $R_{m, k}$ is the smallest closed rectangle which contains the cone $\left\{r \theta: \theta \in D_{m, k},-2^{m} \leq r \leq 2^{m}\right\}$. Note that $R_{m, k}$ is uniquely determined by $D_{m, k}$ up to rotation about its major axis, and any choice will do. Then $\left|R_{m, k}\right| \approx 2^{d m}\left|D_{m, k}\right|$ uniformly in $m$ and $k$, with constants of comparability depending only on $d$, and therefore

$$
\sum_{k}\left|R_{m, k}\right| \approx 2^{d m} \sum_{k}\left|D_{m, k}\right| \approx\left|S_{m}\right|, \quad m \geq 1,
$$

with similar constants. Also

$$
S_{m} \subseteq\left\{r \theta: \theta \in \Theta_{m}, 0 \leq r \leq 2^{m}\right\} \subseteq \bigcup_{k} R_{m, k},
$$

which verifies (4.1).

We will now prove Theorems 1.3, 1.4, and 1.5. 
Proof of Theorem 1.3. Let us begin by showing how Theorem 1.3 follows from Theorem 1.5, or from Theorem 1.4 if $p=2$. In fact, condition (1.4) means that

$$
\left(\frac{1}{|R|} \int_{R} w(x) d x\right)^{1 / p}\left(\frac{1}{|R|} \int_{R} w(x)^{-p^{\prime} / p} d x\right)^{1 / p^{\prime}} \leq c
$$

for $R \in \mathcal{B}\left(R_{m, k}\right)$ for all $k, m$, with $c$ independent of $R, k$ and $m$. This leads by standard methods to the existence of $r>1$ and $C>0$ independent of $R, k$ and $m$ such that

$$
\left(\frac{1}{|R|} \int_{R} w(x)^{r} d x\right)^{1 / r p}\left(\frac{1}{|R|} \int_{R} w(x)^{-r p^{\prime} / p} d x\right)^{1 / r p^{\prime}} \leq C
$$

for $R \in \mathcal{B}\left(R_{m, k}\right)$. To be more precise on this point, when (4.2) holds uniformly for all cubes, then the "reverse Hölder inequality" argument shows that (4.3) holds for all cubes with constants $r>1$ and $C>0$ which depend only on $c$ and the dimension. However, this argument is invariant under scaling and applies upon replacing cubes with $R \in \mathcal{B}\left(R_{m, k}\right)$ for each $m, k$, with constants depending only on $d$ and the constant $c$ in (4.2). From (4.3) we see that conditions (1.9) and (1.10) hold with $c_{m, k}$ chosen to be $C\left|R_{m, k}\right|$. Moreover, assuming that $\Omega \in L \log L\left(\mathbf{S}^{d-1}\right)$, we also have that (1.8) holds, since

$$
\begin{aligned}
\sum_{\substack{m \geq 0 \\
k}}(m+1) c_{m, k} & \leq c \sum_{m \geq 0}(m+1)\left|S_{m}\right| \quad \text { by }(4.1) \\
& \leq c\|\Omega\|_{L \log L\left(\mathbf{S}^{d-1}\right)}
\end{aligned}
$$

as we have already observed in the proof of Theorem 1.2. The first statement in Theorem 1.3 now follows from Theorem 1.5, and the second statement in Theorem 1.3 follows by combining the first statement with Theorem 1.2.

Proof of Theorem 1.4. We claim that if $1<p<\infty$ and $w$ satisfies (1.7) and (1.8) holds, then

$$
\left\|\left(\sum_{j}\left|G_{j} f_{j}\right|^{p}\right)^{1 / p}\right\|_{p, w} \leq c\left\|\left(\sum_{j}\left|f_{j}\right|^{p}\right)^{1 / p}\right\|_{p, w}
$$

with $c$ independent of $\left\{f_{j}\right\}$. When $p=2$, this is the same as (1.4), and then Theorem 1.4 follows immediately from Theorem 1.2.

In order to prove (4.4), we have only to show that

$$
\left\|G_{j} f\right\|_{p, w} \leq c\|f\|_{p, w}
$$

with $c$ independent of $j$ and $f$. Let $A_{+t}, A_{+t}^{m}$ be the positive operators defined by dropping $\operatorname{sgn} \Omega$ from the definitions of $A_{t}, A_{t}^{m}$, respectively, that is,

$$
\begin{aligned}
& A_{+t} f(x)=\frac{1}{t^{d}} \int_{t S} f(x-y) d y=t^{-d} \chi_{t S} * f(x), \\
& A_{+t}^{m} f(x)=\frac{1}{t^{d}} \int_{t S_{m}} f(x-y) d y=t^{-d} \chi_{t S_{m}} * f(x) .
\end{aligned}
$$


We have (see (1.3))

$$
\begin{aligned}
\left|G_{j} f(x)\right| & =\left|\sum_{m \geq 0} \int_{2^{j-m-1}}^{2^{j+(N-1) m}} A_{t}^{m} f(x) \frac{d t}{t}\right| \\
& \leq \sum_{m \geq 0} \int_{2^{j-m-1}}^{2^{j+(N-1) m}} A_{+t}^{m}(|f|)(x) \frac{d t}{t} .
\end{aligned}
$$

For a stratified starlike cover $\left\{R_{m, k}\right\}_{m, k}$, let

$$
A_{+t}^{m, k} f(x)=\frac{1}{t^{d}} \int_{t R_{m, k}} f(x-y) d y .
$$

Since $S_{m} \subseteq \bigcup_{k} R_{m, k}$, then by Minkowski's inequality,

$$
\left\|G_{j} f\right\|_{p, w} \leq \sum_{m \geq 0} \int_{2^{j-m-1}}^{2^{j+(N-1) m}} \sum_{k}\left\|A_{+t}^{m, k}(|f|)\right\|_{p, w} \frac{d t}{t},
$$

and by Hölder's inequality, $A_{+t}^{m, k}(|f|) \leq A_{+t}^{m, k}\left(|f|^{p} w\right)^{1 / p} A_{+t}^{m, k}\left(w^{-p^{\prime} / p}\right)^{1 / p^{\prime}}$, so

$$
\begin{aligned}
& \left\|A_{+t}^{m, k}(|f|)\right\|_{p, w}^{p} \\
& \leq \frac{1}{t^{d p}} \int_{\mathbf{R}^{d}}\left(\int_{x-t R_{m, k}}|f(y)|^{p} w(y) d y\right)\left(\int_{x-t R_{m, k}} w(z)^{-p^{\prime} / p} d z\right)^{p / p^{\prime}} w(x) d x \\
& \quad=\frac{1}{t^{d p}} \int_{\mathbf{R}^{d}}|f(y)|^{p} w(y)\left\{\int_{y+t R_{m, k}} w(x)\left(\int_{x-t R_{m, k}} w(z)^{-p^{\prime} / p} d z\right)^{p / p^{\prime}} d x\right\} d y .
\end{aligned}
$$

If $z \in x-t R_{m, k}$ and $x \in y+t R_{m, k}$, then $z \in y+t R_{m, k}-t R_{m, k} \subseteq y+2 t R_{m, k}$. Thus the last expression is at most

$$
\begin{aligned}
\frac{1}{t^{d p}} \int_{\mathbf{R}^{d}}|f(y)|^{p} w(y)\left\{( \int _ { y + 2 t R _ { m , k } } w ( x ) d x ) \left(\int_{y+2 t R_{m, k}}\right.\right. & \left.\left.w(z)^{-p^{\prime} / p} d z\right)^{p / p^{\prime}}\right\} d y \\
& \leq\|f\|_{p, w}^{p}\left(\frac{\left|2 t R_{m, k}\right| c_{m, k}}{t^{d}\left|R_{m, k}\right|}\right)^{p} \\
& =\left(2^{d} c_{m, k}\right)^{p}\|f\|_{p, w}^{p},
\end{aligned}
$$

assuming that (1.7) holds. Collecting estimates, we obtain

$$
\begin{aligned}
\left\|G_{j} f\right\|_{p, w} & \leq 2^{d} \sum_{m \geq 0} \int_{2^{j-m-1}}^{2^{j+(N-1) m}} \frac{d t}{t} \sum_{k} c_{m, k}\|f\|_{p, w} \\
& =\left(2^{d} \ln 2\right) \sum_{m \geq 0}(N m+1) \sum_{k} c_{m, k}\|f\|_{p, w} \\
& =c\|f\|_{p, w} \quad \text { by }(1.8),
\end{aligned}
$$

with a constant $c$ depending on $N$, but independent of $j$ and $f$. This proves our claim, and so also Theorem 1.4. 
Proof of Theorem 1.5. We first consider the case when $1<p \leq 2$. Let $G$ be the maximal operator defined by

$$
G f(x)=\sum_{m \geq 0}(m+1) \sup _{t>0} A_{+t}^{m}(|f|)(x) .
$$

We have

Lemma 4.1. For all $j, x$ and $f$,

$$
\left|G_{j} f(x)\right| \leq(N \ln 2) G f(x),
$$

where $N$ is the constant appearing in the definition of the operators $G_{j}$.

Also, $G$ satisfies the following Hölder type inequalities:

$$
\begin{aligned}
G(f g) & \leq\left\{G\left(|f|^{r}\right)\right\}^{1 / r}\left\{G\left(|g|^{r^{\prime}}\right)\right\}^{1 / r^{\prime}}, \\
G f & \leq D^{1-1 / r}\left\{G\left(|f|^{r}\right)\right\}^{1 / r},
\end{aligned}
$$

for all $r>1$, where $D=\sum_{m \geq 0}(m+1)\left|S_{m}\right|$.

Proof. By definition of $G_{j}$,

$$
\begin{aligned}
\left|G_{j} f(x)\right| & \leq \sum_{m \geq 0} \int_{2^{j-m-1}}^{2^{j+(N-1) m}} A_{+t}^{m}(|f|)(x) \frac{d t}{t} \\
& \leq \sum_{m \geq 0} \sup _{t>0} A_{+t}^{m}(|f|)(x) \int_{2^{j-m-1}}^{2^{j+(N-1) m}} \frac{d t}{t} \\
& =\sum_{m \geq 0}(N m+1)(\ln 2) \sup _{t>0} A_{+t}^{m}(|f|)(x) \\
& \leq(N \ln 2) G f(x),
\end{aligned}
$$

which proves the first part of the lemma. For the Hölder type inequalities, Hölder's integral inequality gives $A_{+t}^{m}(f g) \leq\left\{A_{+t}^{m}\left(|f|^{r}\right)\right\}^{1 / r}\left\{A_{+t}^{m}\left(|g|^{r^{\prime}}\right)\right\}^{1 / r^{\prime}}$ pointwise, and used with Hölder's inequality for sums, we have

$$
\begin{aligned}
G(f g)(x) & \leq \sum_{m \geq 0}(m+1) \sup _{t>0}\left\{A_{+t}^{m}\left(|f|^{r}\right)\right\}^{1 / r} \sup _{t>0}\left\{A_{+t}^{m}\left(|g|^{r^{\prime}}\right)\right\}^{1 / r^{\prime}} \\
& \leq\left\{\sum_{m \geq 0}(m+1) \sup _{t>0} A_{+t}^{m}\left(|f|^{r}\right)\right\}^{1 / r}\left\{\sum_{m \geq 0}(m+1) \sup _{t>0} A_{+t}^{m}\left(|g|^{r^{\prime}}\right)\right\}^{1 / r^{\prime}} \\
& =\left\{G\left(|f|^{r}\right)\right\}^{1 / r}\left\{G\left(|g|^{r^{\prime}}\right)\right\}^{1 / r^{\prime}} .
\end{aligned}
$$

Choosing $g=1$ in this inequality gives the second Hölder's inequality of Lemma 4.1 .

The operator $G$ may be analyzed in terms of somewhat simpler operators. For a starlike set $S$, define

$$
\mathrm{M}_{S} f(x)=\sup _{t>0} A_{+t}(|f|)(x) .
$$

This operator is the Hardy-Littlewood maximal operator relative to the starlike set $S$. It is a natural pointwise majorizant of the operators $A_{t}$, and in view of our singular integral representation formula, it is not surprising that $\mathrm{M}_{S}$ plays a role in our analysis of $\mathrm{T}_{\Omega}$. This operator will obtain somewhat greater significance in 
$\S 5$. For now, its interest is primarily related to the study of the operator $G$, which may be written

$$
G f(x)=\sum_{m=0}^{\infty}(m+1) \mathrm{M}_{S_{m}} f(x),
$$

where $\mathrm{M}_{S_{m}}$ is the starlike maximal operator with respect to the set $S_{m}$. In order to estimate $G$, we will use the following result about $\mathrm{M}_{S_{m}}$ from [CWW].

Lemma 4.2. Let $S$ be a set starlike with respect to the origin, $R_{m, k}$ a stratified starlike cover of $S$, and $1<p<\infty$. Given $m \geq 0$, if there exists $r>1$ such that

$$
\left(\frac{1}{|R|} \int_{R} w\right)^{1 / p}\left(\frac{1}{|R|} \int_{R} w^{-r p^{\prime} / p}\right)^{1 / r p^{\prime}} \leq \frac{c_{m, k}}{\left|R_{m, k}\right|}
$$

(cf. (1.9)) holds for all $R \in \mathcal{B}\left(R_{m, k}\right)$ for all $k$, then

$$
\left\|\mathrm{M}_{S_{m}}(|f|)\right\|_{p, w} \leq c\left(\sum_{k} c_{m, k}\right)\|f\|_{p, w}
$$

with $c$ independent of $f, m$, and $w$. If (4.12) holds for some $r>1$ for all $R \in$ $\mathcal{B}\left(R_{m, k}\right)$ for all $m \geq 0$ and all $k$, then

$$
\left\|\mathrm{M}_{S} f\right\|_{p, w} \leq c\left(\sum_{m>0} c_{m, k}\right)\|f\|_{p, w} .
$$

This lemma is a special case of Theorem 1.5(C) of [CWW].We take $\mu=0$ there and consider the case of equal weights, thus giving (4.14); the more specialized result (4.13) is obtained by applying the general result to each starlike set $S_{m}$. (We note that the case $\mu=0$ was accidentally omitted in the statement of Theorem $\mathrm{P}$ of [CWW], p. 242.) This result holds for $1<p<\infty$, as stated above, but we only require it for $1<p \leq 2$, where (4.12) is the same as (1.9).

We can now verify Theorem 1.5 for the case $1<p \leq 2$. By considering $\left\{G_{j}\right\}$ as an operator on the sequence space $L^{p}\left(\ell^{q}, w\right)=\left\{\left\{f_{j}\right\}_{j}:\left\|\left|\left\{f_{j}\right\}\right|_{\ell^{q}}\right\|_{p, w}<\infty\right\}$, where $|\cdot|_{\ell q}$ is the $\ell^{q}$-sequence norm, it then suffices by interpolation in $q$ to show that for $1<p \leq 2,(1.8)$ and (1.9) ensure that

$$
\begin{gathered}
\left\|\left(\sum_{j}\left|G_{j} f_{j}\right|^{p}\right)^{1 / p}\right\|_{p, w} \leq c\left\|\left(\sum_{j}\left|f_{j}\right|^{p}\right)^{1 / p}\right\|_{p, w}, \\
\left\|\sup _{j}\left|G_{j} f_{j}\right|\right\|_{p, w} \leq c\left\|\sup _{j}\left|f_{j}\right|\right\|_{p, w},
\end{gathered}
$$

with $c$ independent of $\left\{f_{j}\right\}$. In fact, this interpolation (which is not needed for $p=2$; only (4.15) is then required) will then imply (1.4), and we will be done by applying Theorems 1.1, 1.2.

We have already shown in the proof of Theorem 1.4 that (4.15) holds if (1.7) and (1.8) hold. We now claim that if $1<p<\infty$ and (4.12) holds for constants satisfying (1.8), then

$$
\|G f\|_{p, w} \leq c\|f\|_{p, w}
$$


Note that since

$$
\begin{aligned}
\sup _{j}\left|G_{j} f_{j}(x)\right| & \leq(N \ln 2) \sup _{j}\left|G f_{j}(x)\right| \quad \text { by Lemma } 4.1 \\
& \leq(N \ln 2) G\left(\sup _{j}\left|f_{j}\right|\right)(x),
\end{aligned}
$$

(4.16) follows by applying (4.17) to the function $f=\sup _{j}\left|f_{j}\right|$.

Let us now show (4.17). By definition of $G$, if $1<p<\infty$ and (4.12) holds, then

$$
\begin{aligned}
\|G f\|_{p, w} & \leq \sum_{m \geq 0}(m+1)\left\|\sup _{t>0} A_{+t}^{m}(|f|)\right\|_{p, w} \\
& \leq \sum_{m \geq 0}(m+1) c \sum_{k} c_{m, k}\|f\|_{p, w} \quad \text { by Lemma } 4.2 \\
& =c^{\prime}\|f\|_{p, w},
\end{aligned}
$$

assuming that (1.8) holds as well. This proves (4.17) and so completes the proof of (4.16) and of Theorem 1.5 for the case $1<p \leq 2$.

We note that (4.17) implies that (4.15) and (4.16) hold with $G_{j}$ replaced by $G$. Another significant consequence of the fact that (4.12) together with (1.8) implies (4.17) is the following, which we shall need later:

Observation 4.3. If $\Omega \in L \log L\left(\mathbf{S}^{d-1}\right)$, then $G$ is bounded on $L^{p}$ for $1<p<\infty$.

To see this, let $w=1$ in (4.17), noting that (4.12) holds for $w=1$ and any $r>1$ with constants $c_{m, k}=\left|R_{m, k}\right|$, so that, as in the proof of Theorem 1.3, the fact that $\Omega \in L \log L$ together with (4.1) gives (1.8). This gives the observation.

When $2 \leq p<\infty$, we will use a duality argument to prove (1.4). If $p \geq 2$,

$$
\left\|\left(\sum_{j}\left|G_{j} f_{j}\right|^{2}\right)^{1 / 2}\right\|_{p, w}=\sup \left|\int \sum_{j} G_{j} f_{j} g_{j}\right|
$$

where the supremum is taken over all sequences $\left\{g_{j}\right\}$ with

$$
\left\|\left(\sum_{j}\left|g_{j}\right|^{2}\right)^{1 / 2}\right\|_{p^{\prime}, w^{-p^{\prime} / p}} \leq 1
$$

By definition of $G_{j}$, we have

$$
\int G_{j} f(x) g(x) d x=\int f(x) G_{j}^{*} g(x) d x
$$

where $G_{j}^{*}$ is the operator adjoint to $G_{j}$, and is obtained by reflecting in the origin and conjugating the convolution kernel of $G_{j}$. Thus,

$$
\left|\int \sum_{j}\left(G_{j} f_{j}\right) g_{j}\right| \leq\left\|\left(\sum_{j}\left|f_{j}\right|^{2}\right)^{1 / 2}\right\|_{p, w}\left\|\left(\sum_{j}\left|G_{j}^{*} g_{j}\right|^{2}\right)^{1 / 2}\right\|_{p^{\prime}, w^{-p^{\prime} / p}}
$$

Note that $G_{j}^{*}$ corresponds to $G_{j}$ with $\overline{\Omega(-\theta)}$ in place of $\Omega(\theta)$, and the corresponding starlike sets are $-S,-S_{m}$. Since the rectangles $R_{m, k}$ were chosen to be symmetric with respect to the origin, these same rectangles also serve as a stratified starlike cover for $-S$. Consequently, since now $1<p^{\prime} \leq 2$, applying our earlier results to the exponent $p^{\prime}$ and the weight $w^{-p^{\prime} / p}$ gives

$$
\left\|\left(\sum_{j}\left|G_{j}^{*} g_{j}\right|^{2}\right)^{1 / 2}\right\|_{p^{\prime}, w^{-p^{\prime} / p}} \leq c\left\|\left(\sum_{j}\left|g_{j}\right|^{2}\right)^{1 / 2}\right\|_{p^{\prime}, w^{-p^{\prime} / p}},
$$


provided that there is an $r>1$ so that (1.10) holds for constants $c_{m, k}$ satisfying (1.8). This is because (1.10) and (1.9) are the same upon interchanging weights $w$, $w^{-p^{\prime} / p}$ and exponents $p, p^{\prime}$. This proves the first part of Theorem 1.5 for $2 \leq p<\infty$, and the second part follows by combining the first part with Theorems 1.1 and 1.2.

It is also possible to derive the second part of Theorem 1.5 in the case $p \geq 2$ directly by duality from the case $1<p \leq 2$.

Proof of Theorem 1.6. A straightforward computation shows that the bound (1.5) holds for the weights in part (B) of the theorem, and so for this part of Theorem 1.6 we see that the square inequality (1.4) holds as a consequence of Theorem 1.3.

It remains to prove part (A) of the theorem. We will give one proof here that is based on results from [W4], which uses different methods to also obtain the conclusion of this part of Theorem 1.6. In $\S 5$ we will give another proof which uses only results developed in this paper. To do this, we require the introduction of yet another maximal operator. For $\Omega$ homogeneous of degree 0 , let

$$
\mathrm{M}_{\Omega} f(x)=\sup _{t>0} \frac{1}{t^{d}} \int_{|y|<t}|f(x-y)||\Omega(y)| d y .
$$

This operator is the homogeneous maximal operator relative to $\Omega$, and in many ways is the operator most appropriate for the study of $\mathrm{T}_{\Omega}$ using the standard singular integral representation. The operators $G, \mathrm{M}_{S}$ and $\mathrm{M}_{\Omega}$ are quite different in their definitions, but they are closely related. For example, as the following result shows, certain comparisons exist between them.

Lemma 4.4. For any $\Omega$, let $S$ be the starlike set associated with $\Omega$. Then

$$
\begin{aligned}
& \mathrm{M}_{S} f(x) \leq G f(x), \\
& \mathrm{M}_{\Omega} f(x) \leq c_{d}\{M f(x)+G f(x)\}
\end{aligned}
$$

for any function $f$ and every $x \in \mathbf{R}^{d}$.

If $\Omega \in L(\log L)^{\gamma}\left(\mathbf{S}^{d-1}\right)$ for each $\gamma>0$, then for each $\lambda>1$, we also have

$$
\begin{aligned}
& G f(x) \leq c_{\Omega, \lambda} \mathrm{M}_{S}\left(|f|^{\lambda}\right)(x)^{1 / \lambda}, \\
& G f(x) \leq c_{d} M f(x)+c_{\Omega, \lambda} \mathrm{M}_{\Omega}\left(|f|^{\lambda}\right)(x)^{1 / \lambda}
\end{aligned}
$$

with constants independent of $f, x$.

The degree of integrability of $\Omega$ that this lemma requires is satisfied if $\Omega \in$ $L^{r}\left(\mathbf{S}^{d-1}\right)$ for some $r>1$ (which is the integrability requirement of part (A) of Theorem 1.6). Except for Theorem 1.6, this is a stronger requirement than has appeared so far in this paper, and this integrability requirement will also be significant in the following section.

Proof of Lemma 4.4. The first inequality in (4.18) is easy since

$$
\mathrm{M}_{S} f \leq \sum_{m \geq 0} \mathrm{M}_{S_{m}} f \leq \sum_{m \geq 0}(m+1) \mathrm{M}_{S_{m}} f=G f .
$$

To see the first inequality in (4.19), for $\lambda>1$, let

$$
c_{\lambda}=\left(\sum_{m \geq 0}(m+1)^{2 \lambda^{\prime}}\left|S_{m}\right|\right)^{1 / \lambda^{\prime}},
$$


and observe this constant is finite because $\Omega \in L(\log L)^{2 \lambda^{\prime}}$. The sum of the harmonic series of order $\lambda, h_{\lambda}=\sum_{m \geq 0}(m+1)^{-\lambda}$, is of course also finite. Using Hölder's inequality for integrals and sums with exponents $\lambda, \lambda^{\prime}$, and also observing that $A_{+t}^{m}(|f|) \leq A_{+t}(|f|)$ a.e., we see that

$$
\begin{aligned}
G f & =\sum_{m \geq 0}(m+1) \sup _{t>0} A_{+t}^{m}(|f|) \\
& \leq \sum_{m \geq 0}(m+1)^{2}\left|S_{m}\right|^{1 / \lambda^{\prime}}(m+1)^{-1}\left\{\sup _{t>0} A_{+t}^{m}\left(|f|^{\lambda}\right)\right\}^{1 / \lambda} \\
& \leq\left\{\sum_{m \geq 0}(m+1)^{2 \lambda^{\prime}}\left|S_{m}\right|\right\}^{1 / \lambda^{\prime}}\left\{\sum_{m \geq 0} \frac{1}{(m+1)^{\lambda}} \sup _{t>0} A_{+t}^{m}\left(|f|^{\lambda}\right)\right\}^{1 / \lambda} \\
& \leq c_{\lambda} h_{\lambda}^{1 / \lambda} \mathrm{M}_{S}\left(|f|^{\lambda}\right)^{1 / \lambda},
\end{aligned}
$$

giving the first inequality in (4.19).

To prove the second inequality in (4.18), recall that for $m \geq 1,2^{m-1} \leq \rho(\theta)=$ $|\Omega(\theta)|^{1 / d} \leq 2^{m}$ for $\theta \in \Theta_{m}$, so that for $m \geq 1$ and $s=t / 2^{m-1}$,

$$
\begin{aligned}
\frac{1}{t^{d}} \int_{|y|<t}|f(x-y)||\Omega(y)| \chi_{\Theta_{m}}\left(\frac{y}{|y|}\right) d y & \leq \frac{2^{m d}}{t^{d}} \int_{\Theta_{m}} \int_{0}^{t \rho(\theta) / 2^{m-1}}|f(x-r \theta)| r^{d-1} d r d \theta \\
& =2^{d} A_{+s}^{m}(|f|)(x) .
\end{aligned}
$$

Thus,

$$
\begin{aligned}
\mathrm{M}_{\Omega} f(x) & \leq \sup _{t>0} \frac{1}{t^{d}} \int_{|y|<t}|f(x-y)| d y+\sum_{m \geq 1} \sup _{t>0} \frac{1}{t^{d}} \int_{|y|<t}|f(x-y)||\Omega(y)| \chi_{\Theta_{m}}\left(\frac{y}{|y|}\right) d y \\
& \leq c_{d} M f(x)+2^{d} \sum_{m \geq 1} \sup _{t>0} A_{+t}^{m}(|f|)(x) \\
& \leq c_{d}\{M f(x)+G f(x)\},
\end{aligned}
$$

giving the second inequality in (4.18). Similarly, for $m \geq 1$,

$$
\begin{aligned}
A_{+t}^{m}(|f|)(x) & \leq \frac{1}{t^{d}} \int_{\Theta_{m}} \int_{0}^{2^{m} t}|f(x-r \theta)| r^{d-1} d r d \theta \\
& \leq 2^{d} \frac{1}{\left(2^{m} t\right)^{d}} \int_{|y|<2^{m} t}|f(x-y)||\Omega(y)| \chi_{\Theta_{m}}\left(\frac{y}{|y|}\right) d y,
\end{aligned}
$$

and when $f$ is the function which is the constant 1 , the right side of this inequality is $\approx 2^{m d}\left|\Theta_{m}\right| \approx\left|S_{m}\right|$, so using a Hölder's inequality argument like the one which was used to give the first inequality in (4.19), but bounding the $m=0$ term by $M$ as we did above, we get the second inequality in (4.19). This completes the proof of Lemma 4.4.

We use Lemma 4.4 in the following way. In [W4, Theorem 3 and the Corollary to Theorem 2] we find that if $\Omega \in L^{r}\left(\mathbf{S}^{d-1}\right)$ for some $r>1$ and if $p$ and $w$ are as given in any of the cases of part (A) of Theorem 1.6, the maximal operator $\mathrm{M}_{\Omega}$ 
satisfies the vector-valued inequalities

$$
\left\|\left\{\sum_{j}\left|\mathrm{M}_{\Omega} f_{j}\right|^{q}\right\}^{1 / q}\right\|_{p, w} \leq C_{p, q, w}\left\|\left\{\sum_{j}\left|f_{j}\right|^{q}\right\}^{1 / q}\right\|_{p, w},
$$

for all $q>1$. Since $w \in A_{p}$ for these cases, then using (2.22) we see that the corresponding inequalities also hold for the Hardy-Littlewood maximal operator $M$. This then gives square inequalities for $G$, that is,

$$
\left\|\left\{\sum_{j}\left|G f_{j}\right|^{2}\right\}^{1 / 2}\right\|_{p, w} \leq C_{p, w}\left\|\left\{\sum_{j}\left|f_{j}\right|^{2}\right\}^{1 / 2}\right\|_{p, w},
$$

for the same choices of $p$ and $w$, since (4.19) and the triangle inequality can be used to bound the left side of the above inequality by expressions involving $\mathrm{M}_{\Omega}$ and $M$. This gives (4.21) using (4.20) (taking $q=2 / \lambda$ for $\lambda$ close enough to 1 in order that $\left.w \in A_{p / \lambda}\right)$ and the vector-valued inequalities for $M$ in (2.22). Using Lemma 4.1, we then get (1.4) from (4.21), finishing the proof of Theorem 1.6.

\section{Further RESUlts}

In $\S 2$ we discussed two-weight Littlewood-Paley theory which so far we have not used. We use it now in this section to prove various two-weight inequalities for $\mathrm{T}_{\Omega}$. Our principal result is the the following, which may be viewed as a two-weight analogue of Theorem 1.2.

Theorem 5.1. Suppose $\Omega \in L \log L\left(\mathbf{S}^{d-1}\right)$ and has integral 0 on $\mathbf{S}^{d-1}$. Let $1<$ $p<\infty$ and suppose $R$ is a positive sublinear operator such that the square inequality

$$
\int\left\{\sum_{j}\left|G_{j} f_{j}\right|^{2}\right\}^{p / 2} v \leq C_{p, R} \int\left\{\sum_{j}\left|f_{j}\right|^{2}\right\}^{p / 2} R v
$$

holds for all weights $v$. Then for this choice of $p$ and for $r, s>1$, there is a constant $C=C_{p, r, s, N, R}$ such that

$$
\int\left|\mathrm{T}_{\Omega} f\right|^{p} v \leq C \int|f|^{p}\left\{M_{s} v+M_{r} R M_{s} v\right\}
$$

holds for all weights $v$ and for all $f \in \mathcal{S}$.

Proof of Theorem 5.1. In addition to (5.1), the proof also relies upon appropriate two-weight square inequalities for $M$. The inequalities we need are

$$
\left\|\left\{\sum_{j}\left|M f_{j}\right|^{2}\right\}^{1 / 2}\right\|_{p, v} \leq C_{p, r}\left\|\left\{\sum_{j}\left|f_{j}\right|^{2}\right\}^{1 / 2}\right\|_{p, M_{r} v},
$$

for $r>1$ and $1<p<\infty$, where we recall that $M_{r} v=M\left(v^{r}\right)^{1 / r}$. To prove this, first note that it suffices to prove the corresponding inequality with $M_{r} v$ in place of $v$ on the left, since $v \leq M_{r} v$ pointwise a.e. But $M_{r} v$ is an $A_{p}$ weight (in fact an $A_{1}$ weight by (2.16)), which gives (5.3) by (2.22).

Using the two-weight Littlewood-Paley theory in place of the one-weight theory, we now show that the proof of Theorem 5.1 follows from the square inequalities (5.1) and (5.3) in much the same way Theorem 1.2 followed from the square inequality (1.4) and the square inequality contained in (2.22). Since $v \leq M_{s} v$, we see that (5.2) holds if the same inequality, up to a constant multiple, holds for $\mathrm{T}_{\Omega} f+$ $c_{\Omega} f$. Consequently, it suffices by Theorem 1.1 to find the corresponding two-weight inequality for $\int_{0}^{\infty} A_{t} f d t / t$. This operator is split into the terms $I, I I, I I I$ for $N$ to be determined, just as in Theorem 1.2. Again as in the proof of Theorem 1.2, we 
start by assuming the strong cancellation condition (2.29), and we will later make the passage to the more general cancellation requirement.

For $I I$, we use the identity (2.36) to get

$$
\begin{aligned}
\int_{\mathbf{R}^{d}}|I I|^{p} v & \leq C_{p, s} \int_{\mathbf{R}^{d}}\left\{\sum_{j}\left|G_{j} Q_{j}^{2} f\right|^{2}\right\}^{p / 2} M_{s} v \\
& \leq C_{p, s, R} \int_{\mathbf{R}^{d}}\left\{\sum_{j}\left|Q_{j}^{2} f\right|^{2}\right\}^{p / 2} R M_{s} v \\
& \leq C_{p, r, s, R} \int_{\mathbf{R}^{d}}|f|^{p} M_{r} R M_{s} v .
\end{aligned}
$$

Here, the first inequality is the Littlewood-Paley inequality (2.18) for $Q_{j}^{2}$, the second follows by (5.1) (with $M_{s} v$ in place of $v$ ), and the last inequality follows by the Littlewood-Paley inequality (2.17) for $Q_{j}^{2}$. Our arguments for the terms $I, I I I$ make essentially the same use of Littlewood-Paley theory and differ only in the treatment of the intermediate square term.

For the term $I I I$, note that by $(2.16)$, if $0<r<s$, then

$$
M_{r} M_{s} v(x) \leq C_{r, s} M_{s} v(x)
$$

for a.e. $x$ and every $v$. Where we used (2.40) in the one-weight argument, we will now use the following two-weight replacement: for any $\lambda>1$ there is an $\eta>0$ depending on $\lambda$ such that

$$
\left\|\left\{\sum_{j}\left|Q_{j} B_{s+j-m}^{m} f_{j}\right|^{2}\right\}^{1 / 2}\right\|_{p, v} \leq C_{p, \lambda} 2^{d m} 2^{-\eta s}\left\|\left\{\sum_{j}\left|f_{j}\right|^{2}\right\}^{1 / 2}\right\|_{p, M_{\lambda} v} .
$$

To prove this, we first claim that we have the crude inequality

$$
\left\|\left\{\sum_{j}\left|Q_{j} B_{s+j-m}^{m} f_{j}\right|^{2}\right\}^{1 / 2}\right\|_{p, v} \leq C_{p, r} 2^{d m}\left\|\left\{\sum_{j}\left|f_{j}\right|^{2}\right\}^{1 / 2}\right\|_{p, M_{r} M_{s} v}
$$

for exponents $s, r>1$ and for $1<p<\infty$. This is obtained by arguing as in the proof of (2.39), except that we now use the two-weight inequality (5.3) for $M$ in the two places where we used the corresponding one-weight inequality (2.22). By choosing $s>r$ and using (5.4) to simplify terms, we may replace the weight on the right of (5.6) with $M_{s} v$. Next, as in the proof of (2.40), by interpolating with change of measures between the resulting inequality and the good unweighted inequality (2.38) (and replacing $v^{\delta}$ with $v$ ), we then obtain (5.5) for $\lambda>s$.

Using (5.5), we now argue for $I I I$ as in the proof of Theorem 1.2, using the Littlewood-Paley inequalities (2.17), (2.18) in place of (2.14), (2.15) and using (5.5) in place of (2.40). If $N$ is chosen sufficiently large, depending on $\eta$, which in turn depends only on $\lambda$ and $d$, this gives

$$
\int_{\mathbf{R}^{d}}|I I I|^{p} v \leq C_{p, r, s} \int_{\mathbf{R}^{d}}|f|^{p} M_{r} M_{\lambda} M_{s} v .
$$

By choosing $r<\lambda<s$, we may again use (5.4) to collapse terms, and we then get

$$
\int_{\mathbf{R}^{d}}|I I I|^{p} v \leq C_{p, s} \int_{\mathbf{R}^{d}}|f|^{p} M_{s} v .
$$


For $I$, since (2.41) holds, we argue as for (2.42) using the two-weight inequalities for $M$ and the Littlewood-Paley decomposition instead of one-weight inequalities to get

$$
\int_{\mathbf{R}^{d}}|I|^{p} v \leq C_{p, t} \int_{\mathbf{R}^{d}}|f|^{p} M_{r} M_{\lambda} M_{s} v
$$

for $r, s, \lambda>1$, and we can again use (5.4) to simplify the weight.

To make the passage from the strong cancellation requirement to the general case, we again use an argument parallel to the one in the proof of Theorem 1.2. For general $\Omega$ we form the terms $I, I I$ and $I I I$, but with the modified expressions $\widetilde{B}_{j}^{m}$ in place of $B_{j}^{m}$, and we observe that this allows us to obtain essentially the same (i.e., up to a constant multiple) bounds for the modified terms $I$, III that was obtained for the unmodified terms when the strong cancellation condition holds.

For the term $I I$, let $E_{j}$ be the difference between the operator $G_{j}$ defined as a sum of terms $B_{j}^{m}$ and the operator arising from the corresponding sum of modified terms $\widetilde{B}_{j}^{m}$. This operator is given by convolution with a function obtained from sums and averages of the characteristic functions of balls centered at the origin. For our modified $I I$, we have

$$
\begin{aligned}
I I & =\sum_{j} Q_{j}^{2} G_{j} f-\sum_{j} Q_{j}^{2} E_{j} f \\
& =I I_{1}-I I_{2},
\end{aligned}
$$

where $G_{j}$ is the unmodified expression. Then $I I_{1}$ agrees with our original definition of $I I$ and so has the same bound, while for the term $I I_{2}$, we recall from the proof of Theorem 1.2 that $\left|E_{j} f(x)\right| \leq c_{N, \Omega} M f(x)$, so that we may argue essentially as for the term $I$ and obtain a similar bound

$$
\int_{\mathbf{R}^{d}}\left|I I_{2}\right|^{p} v \leq \int_{\mathbf{R}^{d}}|f|^{p} M_{s} v .
$$

This, combined with the bounds for $I I_{1}, I$ and $I I I$, then gives (5.2) for the general case, finishing the proof of Theorem 5.1.

We now will apply Theorem 5.1 to the derivation of various two-weight inequalities. Most of these two-weight inequalities will require additional assumptions about $\Omega$, but the following result does not.

Theorem 5.2. Let $G$ be the operator given by (4.8), and let $G^{*}$ be the corresponding operator formed by replacing $A_{+t}^{m}$ with its adjoint operator $\left(A_{+t}^{m}\right)^{*}$ for each $m$. If $\Omega \in L \log L\left(\mathbf{S}^{d-1}\right)$ and $\Omega$ has integral 0 over $\mathbf{S}^{d-1}$, then for $p \geq 2$ and $r>1$ there is a constant $C_{p, r}$ so that

$$
\int\left|\mathrm{T}_{\Omega} f\right|^{p} v \leq C_{p, r} \int|f|^{p}\left\{M G^{*} M\left(v^{r}\right)\right\}^{1 / r}
$$

for all weights $v$ and all $f \in \mathcal{S}$.

This result is incomplete in that it only gives two-weight inequalities for $p \geq 2$. Its proof can also be used to show that (5.7) holds for $1<p<2$ if $r>p^{\prime}$, but we lack sufficient information about $G_{j}$ and $G^{*}$ to obtain the inequality for $1<p<2$ and $r$ arbitrarily close to 1 .

Proof of Theorem 5.2. We begin with the following result. 
Lemma 5.3. For $1 \leq p<\infty$ and $\Omega \in L \log L$, there is a constant $C_{p, N}$ depending only on $p$ and the parameter $N$ appearing in the definition of $G_{j}$ (see (1.3)) such that

$$
\int\left|G_{j} f\right|^{p} v \leq C_{p, N} \int|f|^{p} G^{*} v
$$

for all weights $v$. For $p \geq 2$ and $r>1$, there is a constant $C_{p, N, r}$ such that

$$
\left\|\left\{\sum_{j}\left|G_{j} f_{j}\right|^{2}\right\}^{1 / 2}\right\|_{p, v} \leq C_{p, N, r}\left\|\left\{\sum_{j}\left|f_{j}\right|^{2}\right\}^{1 / 2}\right\|_{p,\left\{G^{*}\left(v^{r}\right)\right\}^{1 / r}}
$$

for all weights $v$.

Let us assume this lemma for the moment. We will also later show that

$$
M_{r} v \leq D^{-1 / r}\left\{M G^{*} M\left(v^{r}\right)\right\}^{1 / r} \quad \text { a.e., }
$$

for $r \geq 1$, where $D$ is the constant in (4.9). From Theorem 5.1, this inequality and Lemma 5.3 then give (5.7) by the following reasoning: first, by (5.9) we have (5.1) with $R v=\left\{G^{*}\left(v^{r}\right)\right\}^{1 / r}$, and so we can use Theorem 5.1 for this choice of $R$ and for $s=r>1$. In the inequality that results, we then use (5.10) to simplify the weight on the right and so obtain (5.7).

Theorem 5.2 is therefore proven once we show Lemma 5.3 and (5.10). We begin by proving (5.10). In fact we will show that

$$
|f(x)| \leq D^{-1} G^{*} f(x) \quad \text { a.e. }
$$

if $f$ is locally integrable, from which (5.10) easily follows by picking $f=M\left(v^{r}\right)$. Fix $m$ and $f$ and let $x$ be a Lebesgue point of $f$. Then, letting $\widetilde{S}_{m}=-S_{m}$,

$$
\begin{aligned}
\left|f(x)-\frac{1}{\left|t \widetilde{S}_{m}\right|} \int_{t \widetilde{S}_{m}} f(x-y) d y\right| & \leq \frac{1}{\left|t \widetilde{S}_{m}\right|} \int_{t \widetilde{S}_{m}}|f(x-y)-f(x)| d y \\
& \leq \frac{\left|B_{m}\right|}{\left|\widetilde{S}_{m}\right|}\left(\frac{1}{\left|t B_{m}\right|} \int_{t B_{m}}|f(x-y)-f(x)| d y\right),
\end{aligned}
$$

where $B_{m}$ is the ball of radius $2^{m}$ centered at the origin, i.e., the smallest ball with center 0 that contains $\widetilde{S}_{m}$. Since $x$ is a Lebesgue point of $f$, the last expression tends to 0 as $t \rightarrow 0$. Thus

$$
|f(x)|=\left|\lim _{t \rightarrow 0} \frac{1}{\left|t \widetilde{S}_{m}\right|} \int_{t \widetilde{S}_{m}} f(x-y) d y\right| \leq\left|\widetilde{S}_{m}\right|^{-1} \mathrm{M}_{\widetilde{S}_{m}} f(x) .
$$

Multiplying by $(m+1)\left|\widetilde{S}_{m}\right|$ and adding over $m$, we obtain

$$
\left(\sum_{m=0}^{\infty}(m+1)\left|\widetilde{S}_{m}\right|\right)|f(x)| \leq \sum_{m=0}^{\infty}(m+1) \mathrm{M}_{\widetilde{S}_{m}} f(x),
$$

i.e., $D|f(x)| \leq G^{*} f(x)$, as desired. It remains to prove the lemma.

Proof of Lemma 5.3. To show (5.8), we first show that the case $p=1$ follows by duality and Lemma 4.1. Given a measurable function $f$ and an integer $j$, if $h=\operatorname{sgn} G_{j} f$, then

$$
\int_{\mathbf{R}^{d}}\left|G_{j} f\right| v=\int_{\mathbf{R}^{d}}\left(G_{j} f\right) h v=\int_{\mathbf{R}^{d}} f G_{j}^{*}(h v) \leq C_{N} \int_{\mathbf{R}^{d}}|f| G^{*} v .
$$


Next, since the operator $G_{j}$ is given by convolution with a function whose $L^{1}$ norm $C_{N, \Omega}$ is independent of $j$ (see the remarks following (2.28)), then $\left\|G_{j} f\right\|_{\infty} \leq$ $C_{N, \Omega}\|f\|_{\infty}$, and interpolating between $p=1$ and $p=\infty$ gives (5.8) for all $p$.

To show (5.9), first take $p=2$ and observe that interchanging the order of summation and integration and using (5.8) gives

$$
\int_{\mathbf{R}^{d}} \sum_{j}\left|G_{j} f_{j}\right|^{2} v \leq C_{2, N} \int_{\mathbf{R}^{d}} \sum_{j}\left|f_{j}\right|^{2} G^{*} v .
$$

But, as noted before, (4.9) gives $G^{*} v \leq D^{1-1 / r}\left\{G^{*}\left(v^{r}\right)\right\}^{1 / r}$ for any $r>1$, so (5.9) holds if $p=2$. For $p>2$, given $\left\{f_{j}\right\}$ and $v$, we can find a nonnegative $g \in L^{(p / 2)^{\prime}}$ with $\|g\|_{(p / 2)^{\prime}}=1$ for which

$$
\left(\int_{\mathbf{R}^{d}}\left\{\sum_{j}\left|G_{j} f_{j}\right|^{2}\right\}^{p / 2} v\right)^{2 / p}=\int_{\mathbf{R}^{d}} \sum_{j}\left|G_{j} f_{j}\right|^{2} v^{2 / p} g .
$$

We use (5.8) for $p=2$ as above, followed by Hölder's inequality with exponents $\mathrm{pr} / 2,(\mathrm{pr} / 2)^{\prime}$ for our choice of $r>1$ to bound the right side of this expression by

$$
C_{2, N} \int \sum_{j}\left|f_{j}\right|^{2} G^{*}\left(v^{2 / p} g\right) \leq C_{2, N} \int \sum_{j}\left|f_{j}\right|^{2} G^{*}\left(v^{r}\right)^{2 / p r} G^{*}\left(g^{(p r / 2)^{\prime}}\right)^{1 /(p r / 2)^{\prime}} .
$$

Observation 4.3 tells us that $G$ is bounded on $L^{p}$ for $p>1$, and reflecting in the origin tells us that the same is true for $G^{*}$. Hence, since $(\mathrm{pr} / 2)^{\prime}<(p / 2)^{\prime}$,

$$
\left\|G^{*}\left(g^{(p r / 2)^{\prime}}\right)^{1 /(p r / 2)^{\prime}}\right\|_{(p / 2)^{\prime}} \leq C_{p, r}\|g\|_{(p / 2)^{\prime}}=C_{p, r},
$$

and using this with Hölder's inequality in the previous bound gives (5.9).

Note. The only reason we require $p \geq 2$ in Theorem 5.2 is because it is only in this range that we are able to show the square inequality (5.9). In order to use the argument corresponding to the derivation of (5.3) for $1<p<2$, we would need an appropriate two-weight inequality for $G$.

We have so far considered only those inequalities that we are able to prove in the minimal integrability case. By assuming a greater degree of integrability of $\Omega$, we can prove (5.7) for $1<p<2$, as well as some other inequalities of interest. These will involve the maximal operators $\mathrm{M}_{S}$ and $\mathrm{M}_{\Omega}$ defined in $\S 4$. The degree of integrability we assume is the amount appearing in Lemma 4.4.

Theorem 5.4. Suppose $\Omega \in L(\log L)^{\gamma}\left(\mathbf{S}^{d-1}\right)$ for each $\gamma>0$ and that $\Omega$ has integral 0 . Then (5.7) holds for $1<p<\infty$ and $r>1$.

Also, for $1<p<\infty$ and $r>1, \mathrm{~T}_{\Omega}$ satisfies the two-weight inequalities

$$
\begin{gathered}
\int_{\mathbf{R}^{d}}\left|\mathrm{~T}_{\Omega} f\right|^{p} v \leq C_{p, r} \int_{\mathbf{R}^{d}}|f|^{p}\left\{M \mathrm{M}_{-S} M\left(v^{r}\right)\right\}^{1 / r}, \\
\int_{\mathbf{R}^{d}}\left|\mathrm{~T}_{\Omega} f\right|^{p} v \leq C_{p, r} \int_{\mathbf{R}^{d}}|f|^{p}\left\{M \mathrm{M}_{\widetilde{\Omega}} M\left(v^{r}\right)\right\}^{1 / r},
\end{gathered}
$$

for all weights $v$, where $S$ is the starlike set associated with $\Omega$, and $\widetilde{\Omega}(x)=\overline{\Omega(-x)}$.

Proof of Theorem 5.4. Using Lemma 4.4, (or rather its analogue for $G^{*}, \mathrm{M}_{-S}$ and $\mathrm{M}_{\tilde{\Omega}}$ ) we see that (5.11) holds for some choice of $p, r>1$ whenever (5.7) holds for the same $p$ and a lesser choice of $r>1$. The same is also true for (5.12), but the 
reasoning is more intricate. If (5.7) holds for some $p, r>1$, then from Lemma 4.4 we get, for $\lambda>1$,

$$
\int\left|\mathrm{T}_{\Omega} f\right|^{p} v \leq C_{p, r} \int|f|^{p}\left\{M M M\left(v^{r}\right)+\left[M \mathrm{M}_{\widetilde{\Omega}} M\left(v^{r \lambda}\right)\right]^{1 / \lambda}\right\}^{1 / r} .
$$

First, by Hölder's inequality and multiple use of (2.16), we have $\left\{M M M\left(v^{r}\right)\right\}^{1 / r} \leq$ $c_{r} M_{r \lambda} v$ for $\lambda>1$. We claim that for any function $f \in L_{\mathrm{Loc}}^{1}, \Omega \in L^{1}\left(\mathbf{S}^{d-1}\right)$ and $\tau \geq 1$, we have

$$
|f(x)| \leq c_{d, \Omega, \tau} \mathrm{M}_{\Omega}\left(|f|^{\tau}\right)(x)^{1 / \tau} \quad \text { a.e. }
$$

Assuming this for the moment, we obtain (using $f=\mathrm{M}_{r \lambda} v$ and $\tau=r \lambda$ )

$$
M_{r \lambda} v \leq c\left[\mathrm{M}_{\widetilde{\Omega}} M\left(v^{r \lambda}\right)\right]^{1 / r \lambda} \leq c\left[M \mathrm{M}_{\widetilde{\Omega}} M\left(v^{r \lambda}\right)\right]^{1 / r \lambda} \quad \text { a.e. }
$$

so that in (5.13) we may replace the weight on the right side with the weight $\left\{M \mathrm{M}_{\widetilde{\Omega}} M\left(v^{r \lambda}\right)\right\}^{1 / r \lambda}$, for $r, \lambda>1$, which gives (5.12).

To prove the claimed inequality, it is enough to prove the case $\tau=1$ as the case $\tau>1$ then follows by Hölder's inequality, which can be seen to give $\mathrm{M}_{\Omega}(f) \leq$ $\|\Omega\|_{1}^{1-1 / \tau} \mathrm{M}_{\Omega}\left(|f|^{\tau}\right)^{1 / \tau}$. For the case $\tau=1$, define $\Omega_{R}(y)=\Omega(y)$ if $|\Omega(y)| \leq R$ and $\Omega_{R}(y)=0$ otherwise. Let $B_{t}$ be the ball of radius $t$ centered at 0 . Then if we define

$$
c_{t}=\frac{1}{\left|B_{t}\right|} \int_{B_{t}}\left|\Omega_{R}(y)\right| d y,
$$

we see whenever $x$ is a Lebesgue point of $f$ that

$$
\begin{aligned}
\left|c_{t} f(x)-\frac{1}{\left|B_{t}\right|} \int_{B_{t}} f(x-y)\right| \Omega_{R}(y)|d y| & \leq \frac{1}{\left|B_{t}\right|} \int_{B_{t}}|f(x-y)-f(x)|\left|\Omega_{R}(y)\right| d y \\
& \leq \frac{R}{\left|B_{t}\right|} \int_{B_{t}}|f(x-y)-f(x)| d y \\
& \rightarrow 0 \quad \text { as } t \rightarrow 0 .
\end{aligned}
$$

Since

$$
c_{t}=\frac{1}{\left|B_{t}\right|} \int_{0}^{t} r^{d-1} d r \int_{\mathbf{S}^{d-1}}\left|\Omega_{R}(\theta)\right| d \theta=c_{d}\left\|\Omega_{R}\right\|_{1},
$$

we obtain a.e.

$$
\begin{aligned}
|f(x)| c_{d}\left\|\Omega_{R}\right\|_{1} & \leq \sup _{t>0} \frac{1}{\left|B_{t}\right|} \int_{B_{t}}|f(x-y)|\left|\Omega_{R}(y)\right| d y \\
& =\mathrm{M}_{\Omega_{R}}(|f|)(x) \leq \mathrm{M}_{\Omega}(|f|)(x) .
\end{aligned}
$$

Now letting $R \rightarrow \infty$, we get the desired estimate

$$
|f(x)| c_{d}\|\Omega\|_{1} \leq \mathrm{M}_{\Omega}(|f|)(x) \quad \text { a.e. }
$$

Consequently, all of Theorem 5.4 follows once we show that (5.7) holds for all $p$, $r>1$. But this is true for $p \geq 2$ by Theorem 5.2 , so it only remains to prove (5.7) for $1<p<2$. We will do this by an inductive argument which recursively improves the exponent $r$. This argument is an adaptation of a bootstrapping argument of [HW]. Specifically, we will show 
Lemma 5.5. If $\Omega \in L(\log L)^{\gamma}\left(\mathbf{S}^{d-1}\right)$ for all $\gamma>0$ and the inequality

$$
\int_{\mathbf{R}^{d}}\left(\mathrm{M}_{S} f\right)^{p} v^{1 / r} \leq C_{p, r} \int_{\mathbf{R}^{d}}|f|^{p}\left\{M G^{*} M v\right\}^{1 / r}
$$

holds for all $f$ and all $v$, for some exponents $p=p_{0}, r=r_{0}$ satisfying $1<p_{0}<2$ and $1<r_{0} \leq \infty$, then (5.14) also holds for all $f$, all $v$, and all $p, r$ satisfying $p_{0}<p<2$ and $r>r_{1}=r_{1, p}$, where $\frac{1}{r_{1, p}}=\frac{1}{r_{0}}+\frac{p}{2}\left(1-\frac{1}{r_{0}}\right)$. Additionally, if $\Omega$ has integral 0 , then also

$$
\int_{\mathbf{R}^{d}}\left|\mathrm{~T}_{\Omega} f\right|^{p} v^{1 / r} \leq C_{p, r} \int_{\mathbf{R}^{d}}|f|^{p}\left\{M G^{*} M v\right\}^{1 / r}
$$

for all $f \in \mathcal{S}, v$ and for $p, r$ in the same range.

If $\Omega \in L \log L\left(\mathbf{S}^{d-1}\right)$, then by (4.18) and Observation 4.3, we know $\mathrm{M}_{S}$ is bounded on $L^{p}$ for all $p>1$, and so we know (5.14) for $r_{0}=\infty$. Consequently, if $\Omega \in L(\log L)^{\gamma}\left(\mathbf{S}^{d-1}\right)$ for all $\gamma>0$, then the lemma give (5.15) and (5.14) for $1<p<2$ and $r>r_{1}=2 / p$, and we can then use the lemma inductively to obtain (5.15) and (5.14) for $1<p<2$ and $r>r_{k}$ defined recursively from $r_{0}=\infty$ by $\frac{1}{r_{k+1}}=\frac{1}{r_{k}}+\frac{p}{2}\left(1-\frac{1}{r_{k}}\right)$. Now, $r_{k} \searrow 1$, so $(5.15)$ holds for any exponent $r>1$. Replacing $v$ with $v^{r}$, we obtain (5.7) for $1<p<2, r>1$, which then gives the remaining part of Theorem 5.4.

Proof of Lemma 5.5. Note that if $\Omega \in L(\log L)^{\gamma}\left(\mathbf{S}^{d-1}\right)$ for all $\gamma>0$ and if $\mathrm{M}_{S}$ satisfies a norm inequality, say

$$
\left\|\mathrm{M}_{S} f\right\|_{p, u} \leq c\|f\|_{p, v}
$$

then by (4.19), $G$ satisfies the norm inequality

$$
\|G f\|_{q, u} \leq c_{q}\|f\|_{q, v}
$$

for $q>p$. Consequently, if the hypothesis of Lemma 5.5 is satisfied, then we also have

$$
\int_{\mathbf{R}^{d}}|G f|^{p} v^{1 / r_{0}} \leq C_{p, p_{0}, r_{0}} \int_{\mathbf{R}^{d}}|f|^{p}\left\{M G^{*} M v\right\}^{1 / r_{0}}
$$

for all $p>p_{0}$, and since $\sup _{j}\left|G_{j} f_{j}\right| \leq c_{N} G\left(\sup _{j}\left|f_{j}\right|\right)$ by Lemma 4.1, then (5.16) gives

$$
\int_{\mathbf{R}^{d}}\left(\sup _{j}\left|G_{j} f_{j}\right|\right)^{p} v^{1 / r_{0}} \leq C \int_{\mathbf{R}^{d}}\left(\sup _{j}\left|f_{j}\right|\right)^{p}\left\{M G^{*} M v\right\}^{1 / r_{0}} .
$$

But by (5.8), interchanging the order of summation and integration gives

$$
\begin{aligned}
\int_{\mathbf{R}^{d}} \sum_{j}\left|G_{j} f_{j}\right|^{p} v & \leq C_{N, p} \int_{\mathbf{R}^{d}} \sum_{j}\left|f_{j}\right|^{p} G^{*} v . \\
& \leq C_{N, p} \int_{\mathbf{R}^{d}} \sum_{j}\left|f_{j}\right|^{p} M G^{*} M v .
\end{aligned}
$$


When $p<2$ we may then interpolate with change of measures (see the comments following Theorem [SW1] in section 2) between (5.17) and (5.18) to get

$$
\int_{\mathbf{R}^{d}}\left\{\sum_{j}\left|G_{j} f_{j}\right|^{2}\right\}^{p / 2} v^{1 / r_{1}} \leq C_{N, p} \int_{\mathbf{R}^{d}}\left\{\sum_{j}\left|f_{j}\right|^{2}\right\}^{p / 2}\left\{M G^{*} M v\right\}^{1 / r_{1}}
$$

for $r_{1}$ as given in the lemma. But (5.19) is the inequality (5.1) of Theorem 5.1 with $R v=\left\{M G^{*} M\left(v^{r_{1}}\right)\right\}^{1 / r_{1}}$ and with $v^{1 / r_{1}}$ in place of $v$, so by Theorem 5.1,

$$
\int\left|\mathrm{T}_{\Omega} f\right|^{p} v^{1 / r_{1}} \leq C^{\prime} \int|f|^{p}\left\{M_{s}\left(v^{1 / r_{1}}\right)+M_{\lambda}\left\{\left[M G^{*} M M_{s / r_{1}}(v)\right]^{1 / r_{1}}\right\}\right\}
$$

if $\lambda, s>1$. Choosing $\lambda<r_{1}$ and $s>r_{1}$, we may use (5.4) and (5.10) to collapse and consolidate terms, giving

$$
\int\left|\mathrm{T}_{\Omega} f\right|^{p} v^{1 / r_{1}} \leq C^{\prime \prime} \int|f|^{p}\left\{M G^{*} M_{s / r_{1}} v\right\}^{1 / r_{1}} .
$$

Replacing $v$ with $v^{r_{1} / s}$ and using Hölder's inequality with exponent $s / r_{1}$ for $G^{*}$ (see (4.9)) and a similar fact for $M$ then gives (5.15) for $r=s>r_{1}$.

To finish the proof of Lemma 5.5, we must show (5.14) for the range of $p, r$ given in the conclusion. We will prove (5.14) by reducing the derivation of the maximal operator inequality to the corresponding inequality for a family of singular integral operators that behave essentially like $\mathrm{T}_{\Omega}$. We start with the elementary observation that $\mathrm{M}_{S}$ can essentially be reduced to a discrete operator which satisfies the same estimates as $\mathrm{M}_{S}$. If $t>0$ and $j$ is such that $2^{j-1} \leq t \leq 2^{j}$, then we easily see that

$$
2^{-d} A_{+2^{j-1}}(|f|) \leq A_{+t}(|f|) \leq 2^{d} A_{+2^{j}}(|f|),
$$

so that

$$
2^{-d} \sup _{j \in \mathbf{Z}}\left|A_{+2^{j}} f\right| \leq \mathrm{M}_{S} f \leq 2^{d} \sup _{j \in \mathbf{Z}} A_{+2^{j}}(|f|) .
$$

Therefore, any norm bounds for $\mathrm{M}_{S}$ are comparable to the corresponding norm bounds for the positive operator

$$
A_{*} f=\sup _{j \in \mathbf{Z}}\left|A_{+2^{j}} f\right| .
$$

Similarly, $G$ and $G^{*}$ are comparable to the corresponding operators having the supremum taken over the sequence $\left\{2^{j}: j \in \mathbf{Z}\right\}$ instead of $\{t>0\}$. We can also describe discrete versions of $\left\{G_{j}\right\}_{j \in \mathbf{Z}}$ by

$$
G_{j}^{d} f(x)=\sum_{\substack{m \geq 0 \\ 0 \leq s \leq N m}} A_{+2^{s+j-m}}^{m} f(x),
$$

but since we defined $G_{j}$ in terms of the signed averages $A_{t}$, we do not have comparability between the continuous and discrete versions.

Let $\phi_{j}(x)=2^{-d j} \phi\left(2^{-j} x\right)$ for $\phi$ a smooth, compactly supported, nonnegative function with integral 1 , and define operators

$$
\begin{aligned}
D_{j} f & =A_{+2^{j}} f-|S| \cdot \phi_{j} * f, \\
D_{j}^{m} f & =A_{+2^{j}}^{m} f-\left|S_{m}\right| \cdot \phi_{j} * f .
\end{aligned}
$$

Roughly speaking, the terms $A_{+2^{j}}, A_{+2^{j}}^{m}$ correspond to $B_{j}, B_{j}^{m}$, respectively, and the terms $D_{j}, D_{j}^{m}$ correspond to the terms $\widetilde{B}_{j} \widetilde{B}_{j}^{m}$, respectively, which we used in the general cancellation case. Specifically, $D_{j}, D_{j}^{m}$ annihilate constants, and in 
fact satisfy essentially (i.e., up to a constant multiple) the same size and Fourier transform conditions, respectively, as do the terms $\widetilde{B}_{j}$ and $\widetilde{B}_{j}^{m}$ from the proof of Theorem 1.2. Consequently, by repeating all of our earlier arguments for the general cancellation case (and observing that $G_{j}^{d}$ now occurs everywhere in place of $G_{j}$ ), we see that the singular integral operator

$$
T f=\sum_{j \in \mathbf{Z}} \pm D_{j} f=\sum_{j \in \mathbf{Z}} \pm \sum_{m \geq 0} D_{j}^{m} f
$$

satisfies (up to a constant multiple) the norm inequality (5.15), with operator norm which is bounded by a constant independent of the choice of signs \pm . By a Rademacher function argument, the same norm inequality (up to a constant multiple) is also satisfied by the operator

$$
\mathfrak{S} f(x)=\left\{\sum_{j \in \mathbf{Z}}\left|D_{j} f(x)\right|^{2}\right\}^{1 / 2} .
$$

But since $\sup _{j}\left|\phi_{j} * f\right| \leq c M f$ and $\sup _{j}\left|D_{j} f\right| \leq \mathfrak{S} f$, the triangle inequality gives

$$
\mathrm{M}_{S} f \leq \mathfrak{S} f+c M f
$$

and as $M$ satisfies the norm inequality in question, then so does $\mathrm{M}_{S}$, that is, (5.14) holds in the desired range. This finishes the proof of Lemma 5.5 and Theorem 5.4 .

The idea used above of deriving norm inequalities for $\mathrm{M}_{S}$ from corresponding inequalities for singular integrals resembling $\mathrm{T}_{\Omega}$ can also be applied to our earlier singular integral results. The consequences are sumarized in the following result, which is of interest in its own right.

Theorem 5.6. If we no longer require $\int_{\mathbf{S}^{d-1}} \Omega=0$ and if we use the discrete operators $\left\{G_{j}^{d}\right\}$ in place of $\left\{G_{j}\right\}$, then Theorems 1.2-1.6 and Theorems 5.1, 5.2 also hold (up to a constant multiple) for $\mathrm{M}_{S}$ in place of $\mathrm{T}_{\Omega}$. Wherever $G$ or $G^{*}$ appears we may use either the continuous or discrete version.

Some of the inequalties for $\mathrm{M}_{S}$ which result from this theorem are not as good as ones already proved in $[\mathrm{CWW}]$, but others are new. However, all of the square inequalities resulting from this theorem are new.

Alternative proof of Theorem $1.6(A)$. Throughout the proof we take $1<r \leq \infty$ and $\Omega \in L^{r}\left(\mathbf{S}^{d-1}\right)$ with integral 0 . In order to prove that the square inequality (1.4) holds for the cases (1)-(3) for some given $r$, it suffices to show that it holds for the case $p>r^{\prime}$ and $w \in A_{p / r^{\prime}}$, that is, for all but the endpoint case of (1). This suffices by arguments used in [KW1], which we will only briefly outline. First, the square inequality for the case $p=r^{\prime}$ of (1) follows by interpolation between the inequality for $p>r^{\prime}$ and the unweighted square inequality for $1<p<r^{\prime}$, using the fact that $w^{1+\epsilon} \in A_{p}$ whenever $w \in A_{p}$. Since $T_{\Omega}^{*}$ belongs to the same class of operators, we then get (1.4) for case (2) by duality. Lastly, (1.4) for the cases (1) and (2) together gives (1.4) for the case (3) using Jones' factorization theorem [J] and an interpolation argument, exactly as was used in [KW1], except that now we are interpolating $\ell^{2}$-valued operators.

The same argument, reversing the roles of (1) and (2), shows that for any given $r$ it also suffices to instead prove that (1.4) holds for the case $1<p<r$ and $w^{-p^{\prime} / p} \in A_{p^{\prime} / r^{\prime}}$. 
In order to prove the needed inequalities, we first claim that for all $f$ we have the pointwise inequality

$$
G f(x) \leq C_{\Omega, r, \lambda} M_{\lambda} f(x), \quad \text { for } \lambda>r^{\prime} .
$$

This follows by using Lemma 4.4 and the simple Hölder's inequality estimate

$$
\mathrm{M}_{\Omega} f(x) \leq\|\Omega\|_{r} M_{r^{\prime}} f(x) .
$$

Alternatively, since $S_{m}$ is contained in a ball of radius $2^{m}$, Hölder's inequality gives

$$
M_{S_{m}} f(x) \leq 2^{m d / \lambda}\left|S_{m}\right|^{1-1 / \lambda} M_{\lambda} f(x)
$$

for $\lambda \geq 1$, and since

$$
\begin{aligned}
\left|S_{m}\right| & =d^{-1} \int_{\left\{\theta \in \mathbf{S}^{d-1}: 2^{m-1}<\rho(\theta) \leq 2^{m}\right\}}|\Omega(\theta)| d \theta \\
& \leq \frac{1}{2^{(m-1)(r-1) d}} \int_{\mathbf{S}^{d-1}}|\Omega(\theta)|^{r} d \theta,
\end{aligned}
$$

then if $\lambda>r^{\prime}$ we have

$$
M_{S_{m}} f(x) \leq c_{\Omega, r, \lambda} 2^{-\epsilon m} M_{\lambda} f(x)
$$

for $\epsilon=d[(r-1)(1-1 / \lambda)-1 / \lambda]>0$. This inequality, inserted into the definition of $G$, then gives (5.20).

Using (5.20) and (2.22), we therefore have

$$
\left\|\left\{\sum_{j}\left|G f_{j}\right|^{q}\right\}^{1 / q}\right\|_{p, w} \leq C_{p, q, w}\left\|\left\{\sum_{j}\left|f_{j}\right|^{q}\right\}^{1 / q}\right\|_{p, w}
$$

for $p, q>r^{\prime}$ and $w \in A_{p / r^{\prime}}$ (we first have these inequalities with $\lambda$ in place of $r^{\prime}$, but since we may choose $\lambda$ as close to $r^{\prime}$ as we wish, the by-now familiar open weight argument gives what we have stated). Consequently, if $r>2$ we may take $q=2$ in (5.21) and use Lemma 4.1 to see that (1.4) holds for $p>r^{\prime}$ and $w \in A_{p / r^{\prime}}$, so that we are done by the argument at the beginning of the proof.

It remains to derive the desired square inequalities when $1<r \leq 2$, which we do using Theorem 5.6 and the comments preceding the proof of Lemma 5.5. These give (5.7) for $p>1$ with $\mathrm{M}_{S}$ in place of $\mathrm{T}_{\Omega}$. (We note that the $r$ which appears in (5.7) is different from the present $r$, which is the same as in (5.20).) Since Hölder's inequality and (5.4) show that $M_{s} M v \leq M_{s} M_{s^{\prime}} v \leq c_{s, s^{\prime}} M_{s^{\prime}} v$ for $s^{\prime}>s \geq 1$, then we easily observe from (5.20) and another application of (5.4) that $M G^{*} M v \leq c M_{\lambda} v$ pointwise for $\lambda>r^{\prime}$. Consequently, by Lemma 4.4 we have

$$
\int_{\mathbf{R}^{d}}|G f|^{p} v \leq C \int_{\mathbf{R}^{d}}|f|^{p} M_{\lambda} v, \quad p>1, \lambda>r^{\prime}
$$

for all weights $v$. Since $M_{\lambda}$ is bounded on $L^{p}(w)$ for $p>\lambda$ and $w \in A_{p / \lambda}$, Hölder's inequality applied to the right-hand side of the inequality above gives

$$
\begin{aligned}
\int_{\mathbf{R}^{d}}|G f|^{p} v & \leq C\left\{\int_{\mathbf{R}^{d}}|f|^{p q} w\right\}^{1 / q}\left\{\int_{\mathbf{R}^{d}}\left|M_{\lambda} v\right|^{q^{\prime}} w^{-q^{\prime} / q}\right\}^{1 / q^{\prime}} \\
& \leq C\|f\|_{(p q), w}^{p}\|v\|_{q^{\prime}, w^{-q^{\prime} / q}},
\end{aligned}
$$


provided $q^{\prime}>\lambda$ and $w^{-q^{\prime} / q} \in A_{q^{\prime} / \lambda}$, for any $\lambda>r^{\prime}$. By a duality argument, that is, by taking the supremum over all $v$ with $\|v\|_{q^{\prime}, w^{-q^{\prime} / q}} \leq 1$, we obtain

$$
\int_{\mathbf{R}^{d}}|G f|^{p q} w \leq C \int_{\mathbf{R}^{d}}|f|^{p q} w
$$

for $p>1, q^{\prime}>\lambda>r^{\prime}$ and $w^{-q^{\prime} / q} \in A_{q^{\prime} / \lambda}$. We may choose $p$ as close to 1 and $\lambda$ as close to $r^{\prime}$ as we wish, so by another open-weight argument we get

$$
\int_{\mathbf{R}^{d}}|G f|^{p} w \leq C \int_{\mathbf{R}^{d}}|f|^{p} w, \quad 1<p<r, w^{-p^{\prime} / p} \in A_{p^{\prime} / r^{\prime}} .
$$

This gives the square inequalities for such $p, r$, and $w$ by an earlier argument; i.e., for such $p, r$, and $w$ we have (5.21) for $q=p$ and $q=\infty$ by this inequality and the positivity of $G$, and since $1<p<r \leq 2$, interpolation gives (5.21) for $q=2$. Lemma 4.1 then implies (1.4) for $p, r$, and $w$ as above, and as we noted near the beginning of the proof, this suffices to give (1.4) for all of parts (1)-(3), so we are done.

\section{REFERENCES}

[AJ] K. F. Andersen, R. T. John, Weighted inequalities for vector-valued maximal functions and singular integrals, Studia Math. 69 (1980), 19-31. MR 82b:42015

[CZ] A. P. Calderón and A. Zygmund, On singular integrals, Amer. J. Math, 78 (1956), 289309. MR 18:894a

[CWW] S. Chanillo, D. K. Watson, R. L. Wheeden, Some integral and maximal operators related to starlike sets, Studia Math 107 (1993), 223-255. MR 94j:42027

[CF] R. R. Coifman, C. L. Fefferman, Weighted norm inequalities for maximal functions and singular integrals, Studia Math 51 (1974), 241-250. MR 50:10670

[CorF] A. Cordoba, C. Fefferman, A weighted norm inequality for singular integrals, Studia Mathematica 57 (1976), 97-101. MR 54:8132

[D] J. Duoandikoetxea, Weighted norm inequalities for homogeneous singular integrals, Trans. A. M. S 336 (1993), 869-880. MR 93f: 42030

[DR] J. Duoandikoetxea, J. L. Rubio de Francia, Maximal and singular integral operators via Fourier transform estimates, Inventiones Math 84 (1986), 541-561. MR 87f:42046

[FS] C. Fefferman, E. M. Stein, Some maximal inequalities, American Jour. Math. 93 (1971), 107-115. MR 44:2026

[GR] J. Garcia-Cuerva, J. L. Rubio de Francia, Weighted Norm Inequalities and Related Topics, North Holland, Amsterdam, 1985. MR 87d:42023

[H1] S. Hofmann, Weighted norm inequalities and vector-valued inequalities for certain rough operators, Indiana Jour. Math. 42 (1993), 1-14. MR 94e:42019

[HW] S. Hofmann, D. K. Watson, Erratum to Steve Hofmann's paper, "Weighted norm inequalities and vector-valued inequalities for certain rough operators", to appear.

[J] P. W. Jones, Factorization of $A_{p}$ weights, Ann. of Math. 111 (1980), 511-530. MR 82b: 46035

[K] D. S. Kurtz, Littlewood-Paley and multiplier theorems on weighted $L^{p}$ spaces, Trans. A. M. S. 259 (1980), 235-254. MR 80f: 42013

[KW1] D. S. Kurtz, R. L. Wheeden, Results on weighted norm inequalities for multipliers, Trans. A. M. S. 255 (1979), 343-362. MR 81j:42021

[KW2] A note on singular integrals with weights, Proc. A. M. S. 81 (1981), 391-397. MR 83h:42022

[MW] B. Muckenhoupt, R. L. Wheeden, Weighted norm inequalities for singular and fractional integrals, Trans. A. M. S. 161 (1971), 249-258. MR 44:3155

[S] E. M. Stein, Singular Integrals and Differentiability Properties of Functions, Princeton University Press, Princeton, New Jersey, 1970. MR 44:7280

[SW1] E. M. Stein, G. Weiss, Interpolation of operators with change of measure, Trans. A. M. S. 87 (1958), 159-172. MR 19:1184d 
[SW2] Introduction to Fourier Analysis on Euclidean Spaces, Princeton University Press, Princeton, New Jersey, 1971. MR 46:4102

[T] Hans Triebel, Interpolation Theory, Function Spaces, and Differential Operators, NorthHolland, Amsterdam/New York, 1978. MR 80i:46032b

[W1] D. Watson, Weighted estimates for singular integrals via Fourier transform estimates, Duke Math. Journal 60 (1990), 389-399. MR 91b:42035

[W2] _ 'Dual' weighted bounds for maximal operators from smoothness or Fourier transform estimates, preprint.

[W3] _,$A_{1}$ weights and weak type $(1,1)$ estimates for rough operators, preprint.

[W4] Vector-valued inequalities, factorization, and extrapolation for a family of rough operators, Jour. Funct. Anal. 121 (1994), 389-415. MR 95h:42023

[Z] A. Zygmund, Trigonometric Series, vols. 1 and 2, Cambridge University Press, Cambridge, 1959. MR 21:6498

Department of Mathematics, Rutgers University, New Brunswick, New Jersey 089032101

E-mail address: watsondk@member.ams.org

E-mail address: wheeden@math.rutgers.edu 